\title{
Numerical simulation of water flow around a rigid fishing net
}

\author{
Roger Lewandowski ${ }^{\mathrm{a}}$ and Géraldine Pichot ${ }^{\mathrm{b},{ }^{*}}$
}

\author{
a IRMAR, Campus Beaulieu, Université de Rennes I, 35000 Rennes, France \\ b IFREMER, Technopôle Brest Iroise, 29280 Plouzane, France \\ *: Corresponding author : Tél.: +33 (0)2 232365 46; fax: +33 (0)2 232367 90; Email address: \\ Geraldine.Pichot@ifremer.fr
}

\begin{abstract}
:
This paper is devoted to the simulation of the flow around and inside a rigid axisymmetric net. We describe first how experimental data have been obtained. We show in detail the modelization. The model is based on a Reynolds Averaged Navier-Stokes turbulence model penalized by a term based on the Brinkman law. At the out-boundary of the computational box, we have used a "ghost" boundary condition. We show that the corresponding variational problem has a solution. Then the numerical scheme is given and the paper finishes with numerical simulations compared with the experimental data.
\end{abstract}

Keywords: Fluid mechanics; Turbulence models; Elliptic equations; Variational formulations; Sobolev spaces; Fishing nets 


\title{
Numerical simulation of water flow around a rigid fishing net
}

\author{
Roger Lewandowski ${ }^{a}$, Géraldine Pichot ${ }^{\mathrm{b}, *}$ \\ ${ }^{a}$ IRMAR, Campus Beaulieu, Université de Rennes I, 35000 RENNES, France \\ ${ }^{\mathrm{b}}$ IFREMER, Technopôle Brest Iroise, 29280 PLOUZANE, France
}

\begin{abstract}
This paper is devoted to the simulation of the flow around and inside a rigid axisymmetric net. We describe first how experimental data have been obtained. We show in detail the modelization. The model is based on a Reynolds Averaged NavierStokes turbulence model penalized by a term based on the Brinkman law. At the out-boundary of the computational box, we have used a "ghost" boundary condition. We show that the corresponding variational problem has a solution. Then the numerical scheme is given and the paper finishes with numerical simulations compared with the experimental data.
\end{abstract}

Key words: Fluid mechanics, Turbulence models, Elliptic equations, Variational formulations, Sobolev spaces, fishing nets.

\section{Introduction}

Recent experimental works [3] show that there are less and less fish in the ocean because of intensive industrial fishing. Improvement of the selectivity of fishing nets is a major challenge to preserve fishing resources. There are still too many juvenile fish and fish with no market value are thrown overboard, leading to a real deterioration of the marine ecosystem. Trawls are under study since they are known to be of very poor selectivity. More precisely, we are working on the end part of the trawl, named the cod-end net, which is where most of the selectivity occurs. Now, it appears necessary to quantify the selectivity of existing trawls and also to be able to estimate the benefits of new designs on selectivity in order to motivate their use and to improve them. New designs in trawls are for example cod-end nets with square meshes, with larger mesh sides or with a rigid grid at the proper location...

* Corresponding author. Tél.: +33 (0)2 232365 46; fax: +33 (0)2 23236790

Email address: Geraldine.Pichot@ifremer.fr (Géraldine Pichot). 
Measurements at sea could give some information on selectivity but they are costly, difficult to perform and not easily reproducible (moving net, weather conditions, etc). One cheap way to proceed is to develop a software that simulates the complex catch process so as to perform numerical simulations with different cod-end designs in order to estimate the chance for non-desired fish to escape. To do so, one needs realistic modellings of the net, the surrounding flow and the fish.

The mechanical system made of the elastic net alone in a given laminar uniform flow with very simple interaction laws has been studied already, see for instance in [27], [19] and [24]. A first approach of simulations of the flow around an axisymmetric rigid net has already been performed in [28]. To this point, to our knowledge, no model exists for dealing with the complex question due to the fish. Finally, there is also no work concerning the coupling of an elastic net with the flow. Furthermore, it seems that today the numerical simulation of the complete system net/flow/fish does not exist.

Our present work deals with the flow modelling. It is a necessary step that is motivated by the fact that the local speed has a direct impact on the fish escapement since it determines the catch shape (and then the mesh opening) and the fish tiredness.

In this paper, we deal with the study of the flow around and inside a rigid net in the axisymmetric case. Indeed, the code written in [28] cannot be extended to the fully 3D case. Therefore, the coupling of the deformation of an extensible net with the fluid cannot be considered using this code. Then we have sought a mathematical model that we have tested in the axisymmetric case and that can be extended to the fully 3D case. We have written the corresponding numerical code and performed several simulations to fit the physical constants. Recent investigations have proved already that 3D extension is possible and is currently under progress (see [26]). This allows to believe that it will be possible in the future to couple our fluid code to an elastic code for the net to simulate the system fluid/net.

Our study starts from experiments performed at the IFREMER's tank of Boulognesur-Mer (France). A net model rigidified by a resin (see Fig. 1 below) was built and velocity components were measured during two experimental campaigns. The first one (see [10]) used a Laser Doppler Velocimeter (LDV) technique to get velocity components along different profiles. The second one conducted by the second author of the present paper made use of a Particle Image Velocimeter technique (PIV). This last campaign emphasizes the locations of turbulent structures in the surrounding of the net thanks to instant pictures of the flow. It also gives a good overview of the mean flow by averages of pictures. Concerning the velocity profiles, similar shape were obtained with the two techniques, except slightly lower value with the PIV. In term of accuracy, the LDV technique is much better, that is why the LDV profiles were chosen as the reference experimental data to validate our code, for example see Fig. 15 to 17 at the end of the paper. It is striking how well the experimental velocity data fit with the numerical velocity profiles given by the code. 
The experiments show that the flow we have to simulate is turbulent. Therefore, one needs a turbulent model. Yet, we have done simulations by using only the NavierStokes equations and we did not obtain accurate results. Therefore, we cannot bypass the Turbulent model. We have adapted to the present case a classical RANS one order turbulent closure model (see for instance [15], [17], [16]). It is made of an equation for the turbulent kinetic energy (TKE) and eddy viscosities functions of the TKE into the Navier-Stokes averaged equations. The mixing length has been chosen equal to the local mesh size.

Another important feature of the considered system is that the net behaves like a porous membrane. Taking our inspiration in [1] combined to [2], [14] and [21], we have modeled the net as a porous membrane by penalizing the averaged NavierStokes equation with an additional linear term like in the so-called Brinkman Law. One considers the net as a fictitious domain and one solves the fluid equations in the flow domain as well as in the net domain. However it is an open problem to validate mathematically this part of the modelization by using the homogeneization theory. We only notice that after a right choice of the permeabilty function $K$ (see subsection 3.3) the model yields numerical simulations which fit very well with the experimental data.

The other last important feature of our mathematical model is the boundary conditions at the border of the computational box. On the lateral boundaries, one impose the classical no slip condition. At the incoming boundary, the flow is a given flow. The problem is what to do at the outcoming boundary. The natural and classical boundary condition should be $\boldsymbol{\sigma} \cdot \mathbf{n}=0$ where $\boldsymbol{\sigma}$ is the strain rate tensor. But as observed in [7], one risks artificial eddy reflexions. Moreover, with such a boundary condition we are not able to obtain à priori estimates. To overcome this difficulty, we have adapted the ideas of [7] to the turbulent case. To do this, we have replaced the natural condition by a so-called "ghost condition", the technical condition (8) below. This condition becomes the natural one when the flow is laminar at the incoming and outcoming boundary (see Remark 4.1). Therefore when observing that far from the net the flow remains laminar, we can still take $\boldsymbol{\sigma} \cdot \mathbf{n}=0$ at the outcoming boundary. This is what we did in the numerical simulations. But we stress that the complicated condition (8) is inescapable when dealing with the general mathematical problem.

Our model is given by the system $[(30), \ldots,(38)]$ and the assumptions are summerized by $[(22), \ldots,(29)]$. For the sake of simplicity, we have chosen to study the general mathematical problem in the $2 \mathrm{D}$ case thankfully the axisymmetric case can be easily derived, but technical modifications are necessary (see for instance in [9]). The existence result stated in Theorem 5.1 is our main theoretical contribution in this paper. Uniqueness is an open problem, as well as the general 3D case.

The numerical scheme uses the finite element method in space, an implicit scheme in time for the velocity equation and a semi-implicit scheme for the equation satified by the TKE. The parameters settings are defined in section 6.4. As shown at the 
end of the paper, the numerical results fit remarkably with the experimental datas.

The paper is organized as follows. We start by giving some indications on the experimental framework, then the modelization is described followed by the mathematical analysis. The last part of the paper is devoted to the numerical simulations and the numerical results.

\section{Experimental framework}

Experiments have been carried out at the IFREMER center of Boulogne-sur-Mer. Velocity profiles have been measured inside and around a rigid resin made model built by the Boulogne-Sur-Mer IFREMER team (Fig. 1). This model is like an axisymmetric rigid $1 / 6$ scaled cod-end net with diamond-shaped meshes. The end of the net is filled with a resin mass modelling a one ton catch of fish and trawled with a speed of $1.25 \mathrm{~m} / \mathrm{s}$. The net profile as well as the catch geometry have been derived from an image processing technique.

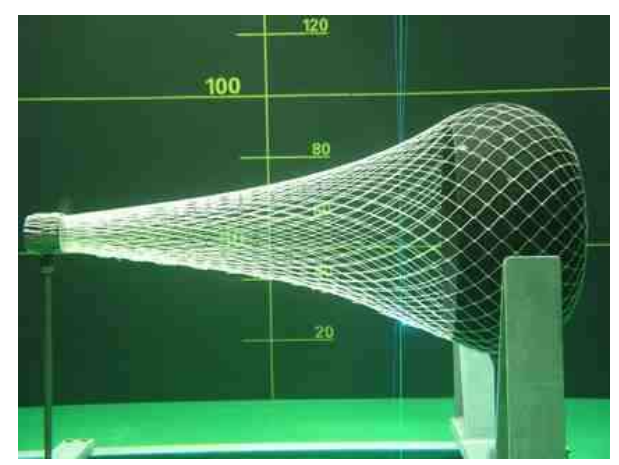

Fig. 1. Model of cod-end net built at IFREMER - Boulogne-sur-Mer

Note that working on a rigid axisymmetric structure excludes accounting for the hydrodynamical forces exterted on the net. Moreover, it restricts the study to an axisymmetric geometry. But, at least measurements are possible and mathematical flow models can be tested.

The model is $1 \mathrm{~m}$ long and has an outer maximal diameter of $0.45 \mathrm{~m}$. It is maintained with a frame and set at the bottom of the IFREMER tank. This tank enables performance of flow measurements with velocities between 0.2 and $2 \mathrm{~m} / \mathrm{s}$. The estimation of the velocity to apply in the tank comes from a Froude similitude yielding an entrance velocity in the tank equal to $0.51 \mathrm{~m} / \mathrm{s}$.

Hydrodynamical measurements have been performed along several profiles (see Fig. $2)$.

One defines a cartesian reference in the tank, the origin being set at the entrance of 


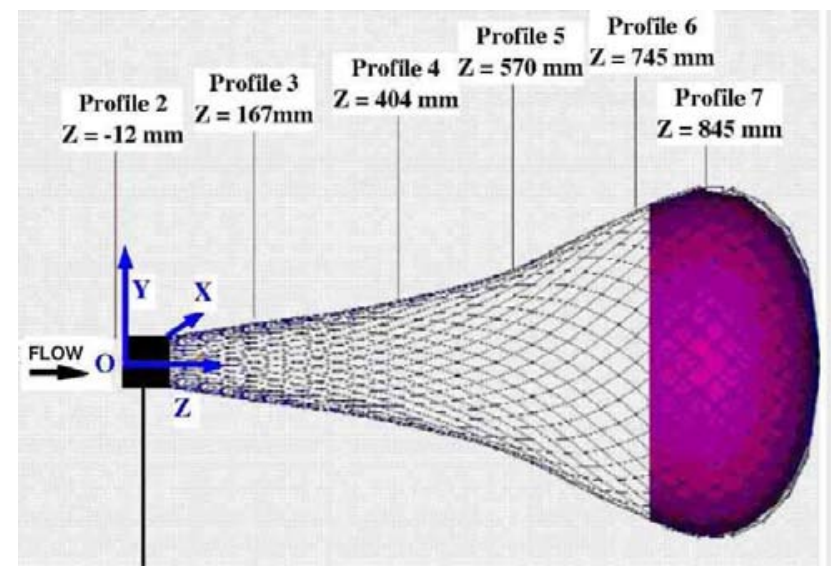

Fig. 2. Profiles considered of the LDV measures

the net.

A Laser Doppler Velocimeter (LDV) technique was used to to collect the $\mathrm{z}$ and $\mathrm{y}$ components of the mean velocity (measures are time averaged). The $\mathrm{z}$ velocity component is the main one we study since it has the direction of the entrance flow, and thus the higher values (see Fig. 3).

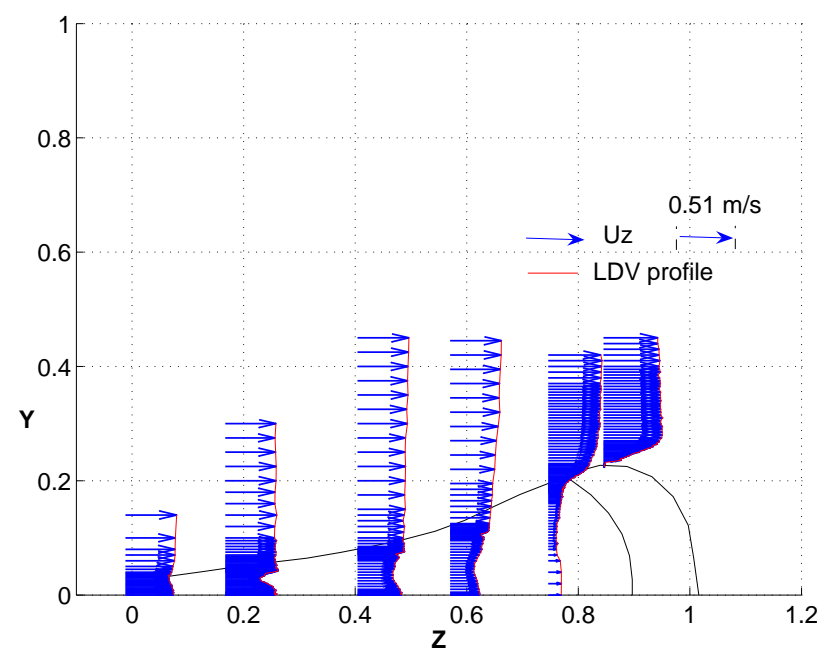

Fig. 3. LDV profiles for the $\mathrm{z}$ component of the velocity.

\section{Modelization}

Our model relies on three features:

(1) Seeing the net, in the fluid point of view, as a porous membrane. The goal is then to define in which manner the fluid is authorized to flow through the net;

(2) Directly taking the net and the catch into account in the averaged Navier-Stokes equations, which leads to averaged Navier-Stokes/Brinkman equations. This 
way, the boundary conditions at the frontiers of the obstacles are implicitely imposed;

(3) Adding a one equation turbulence model to close the system.

Our study deals with the mean flow. One can make the assumption that the mean flow around the net is axisymmetric.

\subsection{Axisymmetric hypothesis}

Assume the cod-end net is embedded in a cylinder full of water. Let us consider an axisymmetric deformation of the net (See Fig. 4). As the net is modeled by a porous membrane the problem reduces to a 2D one, provided an axisymmetric hypothesis of the flow. We admit this hypothesis is a strong one but reasonable in the case of the study of the mean flow, since turbulent structures are smoothed by the averaging.

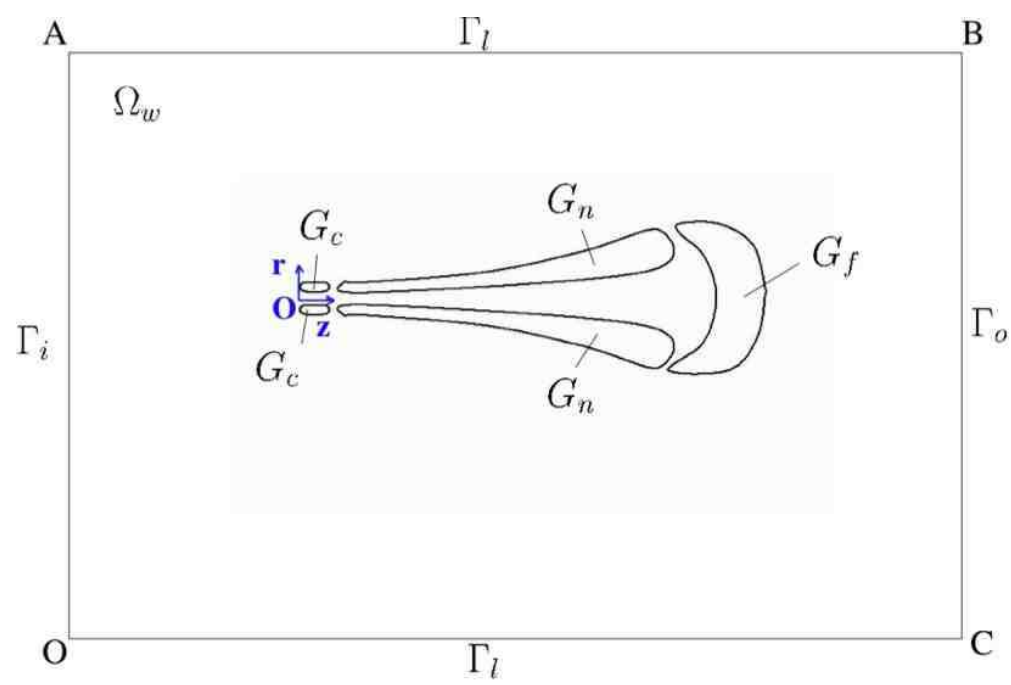

Fig. 4. Geometry and notations

In the following, one notes

- $\Omega_{w}$ the domain occupied by the water,

- $G_{n}$ the net domain,

- $G_{f}$ the the fish domain,

- $G_{c}$ the domain formed by the frame at the entrance of the net model,

$$
\Omega=\Omega_{w} \cup G, \quad G=G_{n} \cup G_{f} \cup G_{c} .
$$

Using the assumption of an axisymmetric flow and the model of an axisymmetric equivalent membrane to describe the net, cylindrical coordinates $(O, r, z, \theta)$ are used in the simulations. At a fixed value of $\theta$, the mathematical problem reduces to a $2 \mathrm{D}$ one. The artificial cylinder reduces to a rectangle in the reference $(O, r, z)$ and the sides of this rectangle are called $\Gamma_{i}, \Gamma_{l}$ and $\Gamma_{o}$ (see Fig. 4). 


\subsection{A membrane model for the net}

Finite elements and finite volumes methods are known to be the common numerical methods to compute fluid dynamics. A mesh is built to discretize the fluid domain. The difficulty of the netting is that it is composed of a great number of meshes. Generating a body-fitted fluid mesh, that is a mesh lying on the nodes and the twines of the net, would be far too complex and computer time consuming. Then, an exact description of the net would be too demanding in computer resources to be conceivable. Another model has to be found.

In the literature, one finds a model of an axisymmetric membrane to deal with an axisymmetric porous structure immersed in a fluid (see [28]).

In [28], the equations are set on the structure location to express a mass transfer in the normal direction to the structure and slip effects in the tangential direction. Then, the tangential velocity, denoted $u_{t}$, is set to be governed by Shaffman's law and the normal velocity, denoted $u_{n}$, by Darcy's law.

This leads to express the velocity components at the wall of the axisymmetric structure by:

$$
\left\{\begin{array}{l}
u_{t}=B \frac{\partial u_{t}}{\partial n}, \\
u_{n}=-K \nabla p
\end{array}\right.
$$

where $n$ is the outer normal of the structure, $p$ the fluid pressure, $K$ a permeability tensor found experimentally, and $B$ a coefficient dependent on the tangential velocity and then deduced from numerical experiences.

To solve the problem, one builts a cartesian mesh from the geometry of the membrane, using curvilinear coordinates. The velocity and pressure unknowns are computed using a finite differences method.

A drawback of this method is that it is based on a cartesian mesh which is not convenient to build and to refine locally in the case of a complex net profile. This work then cannot easily be generalized to the case of a 3D deformation of the net. One has to find a flow model that allows a future coupling with a moving net.

Let us keep the idea of seeing the net as a porous medium, as this assumption has the advantage of making the numerical programming simpler insofar as twines and nodes are no longer taken into account. Then, consider the net and the catch as 
domains with a given permeability.

As shown in Fig. 4, the domain $G_{n}$ delimiting the net has a thickness much larger than the diameter of net twines (which is typically $3 \mathrm{~mm}$ ). This idea actually came from the analysis of the velocity profiles in the $z$ direction obtained by the LDV measurements (see Fig. 3).

One notices on the LDV profiles (see Fig. 3) inner minima of the z velocity component. See Fig. 15-16-17 in the following section for a zoom of each profile. Those minima have been noted down (see Table 1). The inner profile of the membrane has been drawn thanks to those values. The outer profile is in agreement with the profile of the model.

\begin{tabular}{|c||c|c|c|c|}
\hline Profiles & $\begin{array}{c}\text { Profile position } \\
\mathrm{z}[\mathrm{m}]\end{array}$ & $\begin{array}{c}\text { Net location } \\
\text { along } \mathrm{r}[\mathrm{m}]\end{array}$ & $\begin{array}{c}\text { Minimum of } u_{z} \\
\text { Location along } \mathrm{r}[\mathrm{m}]\end{array}$ & $\begin{array}{c}\text { Thickness of the } \\
\text { membrane }[\mathrm{m}]\end{array}$ \\
\hline Profile 3 & 0.167 & 0.045 & 0.027 & 0.018 \\
\hline Profile 4 & 0.404 & 0.0765 & 0.036 & 0.0405 \\
\hline Profile 5 & 0.57 & 0.1125 & 0.045 & 0.0675 \\
\hline Profile 6 & 0.745 & 0.1935 & 0.1035 & 0.09 \\
\hline
\end{tabular}

Table 1

Location of the minima of the $z$ component of the velocity for the considered profiles and thickness of the membrane.

This approach avoids a costly mesh generation. However, it comes with the difficulty of determining which permeability to apply in the different media. The next part is devoted to explain how those media are taken into account directly in the equations.

\subsection{A penalization technique}

The second feature of our model relies on a penalization method that allows us to take the presence of the obstacles into account directly in the fluid equations [14], [21], [2]. The method consists in solving "fluid" equations in the entire domain, even in the net and catch domains. The net domain is seen as a porous medium, and the catch domain as a solid medium, where a no-slip boundary condition should hold. Those media are explicitly included in the fluid equations by the addition of a penality term of the velocity, namely $\frac{1}{K(\mathbf{x})} \mathbf{u}$. This leads to Navier-Stokes/Brinkman equations. Notice that such laws have been derived from an homogeneization process in other situations, as in [1]. This theoretical question remains open in this particular context. The function $K(\mathbf{x})$ varies from one domain to another. It is a permeability parameter that is very small in the solid domains, e.g. the catch, to force the velocity to be zero, and very high in the fluid domain, so that the averaged Navier-Stokes 
equations hold and are set to a defined value or function in the porous domain (here in the net domain) depending on its permeability.

At a first glance, the function $K(\mathbf{x})$ is set to be constant by parts. The net domain is decomposed in three parts, $G_{n}^{i}, \mathrm{i}=1,2,3$ (see Fig. 5) of constant permeability that is all the more important as we are closer to the catch (see Part 6). In a future work, we will try to make it depend on the mesh opening, the mesh angle between the mesh and the local flow.

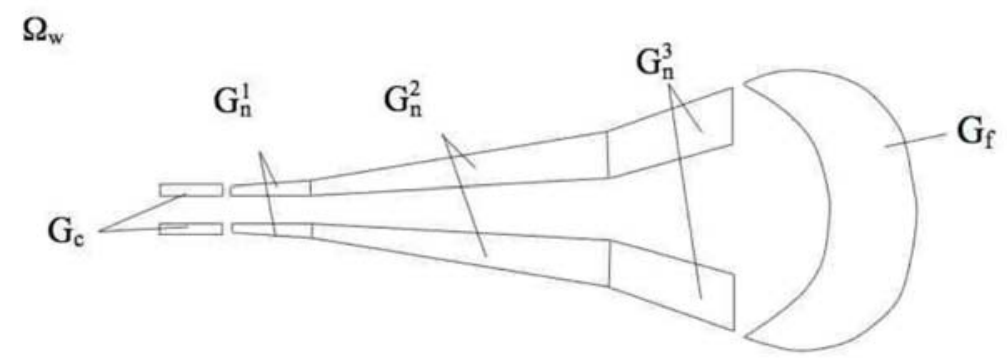

Fig. 5. Decomposition of the net domain - Notations

\subsection{Addition of a turbulence model}

The third point comes with the average of the Navier-Stokes/Brinkman equation, since Direct Numerical Simulation would not be able to treat a problem with such a high Reynolds number (here $R e \cong 10^{5}$, using as reference length the maximal diameter of the catch, i.e. $0.45 \mathrm{~m}$, and the entrance velocity as reference velocity that is equal to $0.51 \mathrm{~m} / \mathrm{s}$ ). A kind of Reynolds Averaged Navier-Stokes (RANS) turbulence model is then added to close the system of equations. It consists of one equation for the turbulent kinetic energy. The averaged NS/Brinkman equation and the turbulent kinetic energy equation are coupled by the means of a eddy viscosity, denoted $\nu_{t}$.

\section{Description of the mathematical problem}

\subsection{The domain}

We return back to the description of the geometry. As already said, the flow under study is axisymmetric. In order to avoid technical complications, we have chosen to study the mathematical problem set in a domain in $\mathbb{R}^{2}$. We refer to [9] to go in further developments in the axisymmetric case.

The boundary $\Gamma$ of the computational box is defined by the input board $\Gamma_{i}$, the 


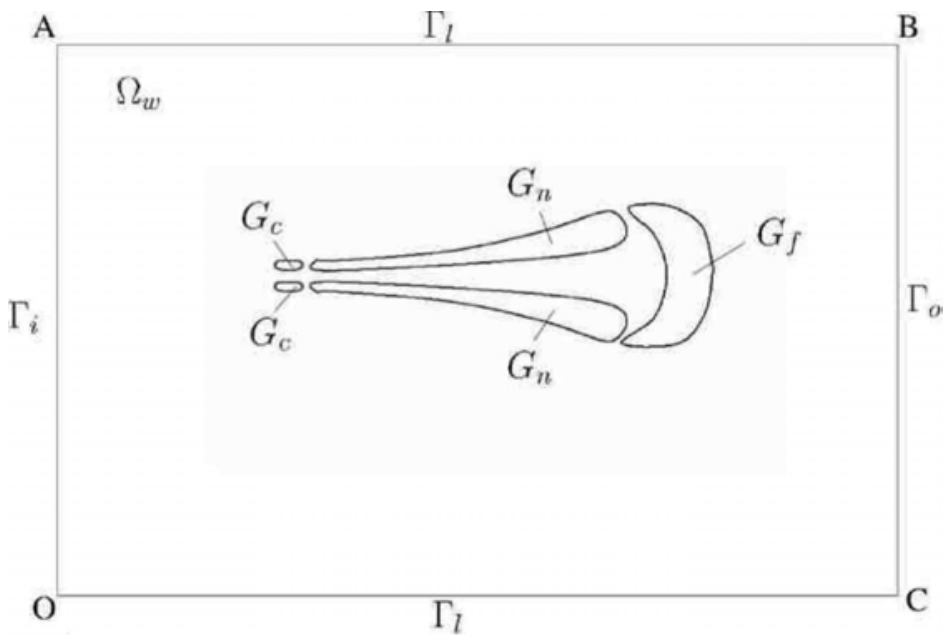

Fig. 6. Description of the domain

lateral boards $\Gamma_{l}$ and the artificial output board $\Gamma_{o}$,

$$
\begin{aligned}
& \Gamma_{i}=[O, A], \quad 0=(0,0), \quad A=(0, \alpha), \\
& \Gamma_{l}=[C, O] \cup[A, B], B=(\beta, \alpha), C=(\beta, 0), \\
& \Gamma_{o}=[B, C], \\
& \Gamma=\Gamma_{i} \cup \Gamma_{l} \cup \Gamma_{o} .
\end{aligned}
$$

\subsection{The equations}

The unknowns are :

- the mean velocity vector field $\mathbf{u}=\mathbf{u}(t, \mathbf{x})=\left(u^{1}(t, \mathbf{x}), u^{2}(t, \mathbf{x})\right), \mathbf{x}=(x, y)$,

- the mean pressure scalar field $p=p(t, \mathbf{x})$,

- the turbulent kinetic energy $k=k(t, \mathbf{x})$

One defines the deformation tensor $\varepsilon$ by

$$
\varepsilon(\mathbf{u})=\frac{\nabla \mathbf{u}+\nabla \mathbf{u}^{T}}{2}
$$

The turbulent strain rate stress tensor $\boldsymbol{\sigma}$ is defined by

$$
\boldsymbol{\sigma}(\mathbf{u}, p, k)=2 \nu_{t}(k, \mathbf{x}) \boldsymbol{\varepsilon}(\mathbf{u})-p \mathbf{I} \mathbf{d} .
$$

The Reynolds Averaged Navier-Stokes turbulent closure model of order one including the Brinkman laws, is given in $[0, T] \times \Omega(T>0)$ by the following equations, where $\varepsilon>0$ is fixed, 


$$
\begin{aligned}
& \partial_{t} \mathbf{u}+(\mathbf{u} \nabla) \mathbf{u}-\nabla \cdot \boldsymbol{\sigma}(\mathbf{u}, p, k)+\left(\frac{1}{\varepsilon}\left(\mathbb{1}_{G_{f} \cup G_{c}}\right)+\frac{1}{K(\mathbf{x})} \mathbb{I}_{G_{n}}+\varepsilon \mathbb{I}_{\Omega_{w}}\right) \mathbf{u}=\mathbf{0}, \\
& \nabla \cdot \mathbf{u}=0 \\
& \partial_{t} k+\mathbf{u} \cdot \nabla k-\nabla \cdot\left(\mu_{t}(k, \mathbf{x}) \nabla k\right)=2 \nu_{t}(k, \mathbf{x})|\varepsilon(\mathbf{u})|^{2}-\mathcal{E}(k, \mathbf{x}) .
\end{aligned}
$$

In the equations above, $\nu_{t}$ and $\mu_{t}$ are the eddy viscosities and $\mathcal{E}$ the backward term. Their analytical expressions are given in section 4.4 below.

\subsection{The boundary conditions and the initial data}

\subsubsection{Boundary conditions}

The input field $\mathbf{u}_{\mathrm{I}}=\left(u_{\mathrm{I}}, 0\right)$ at the boundary $\Gamma_{i}$ is a data of our problem. The boundary conditions we consider are the following.

$$
\begin{aligned}
& \text { on } \Gamma_{i}: \quad \mathbf{u}=\mathbf{u}_{\mathrm{I}}=\left(u_{\mathrm{I}}, 0\right), \quad k=0, \\
& \text { on } \Gamma_{l}: \quad \mathbf{u}=\mathbf{0}, \quad k=0 \text {, } \\
& \text { on } \Gamma_{o}:\left\{\begin{array}{l}
\boldsymbol{\sigma}(\mathbf{u}, p, k) \cdot \mathbf{n}=-\frac{1}{2}(\mathbf{u} . \mathbf{n})^{-}\left(\mathbf{u}-\mathbf{u}_{\mathrm{I}}\right)+(\mathbf{u . n}) \mathbf{u}_{\mathrm{I}} \\
k=0 .
\end{array}\right.
\end{aligned}
$$

In the formulae above, $\mathbf{u}_{\mathrm{I}}$ denotes the field equal to $\left(u_{\mathrm{I}}(x-\beta, 0)\right)$ on $\Gamma_{o}$. One uses the boundary condition (8) in order to avoid eddy reflections at the open boundary $\Gamma_{o}$ and to be able to prove the existence of a dissipative solution to the system (5).

Remark 4.1 The natural boundary condition for the velocity at $\Gamma_{o}$ should be $\boldsymbol{\sigma}(\mathbf{u}, p, k) \cdot \mathbf{n}=\mathbf{0}$. In [7], the authors study the case of the Navier-Stokes equations without a turbulence model and in a channel without a rigid body. They remark that the boundary condition $\sigma \cdot \mathbf{n}=\mathbf{0}$ yields numerical eddy reflexions at the out open boundary. Moreover, the existence of a dissipative solution is not known in such a case because of a term $\int_{\Gamma_{0}}(\mathbf{u} . \mathbf{n})|\mathbf{u}|^{2}$ which appears in the energy equality due to the convection. Without additional information on the sign of (u.n) at $\Gamma_{0}$, no à priori estimate is avaible. This is why the authors in [7] change the boundary conditions. We also change the boundary conditions by an adaptation to the case of our turbulence model. Notice that when the flow is laminar at $\Gamma_{0}$ and $(\mathbf{u . n})>0$ on $\Gamma_{0}$, the boundary condition reduces to the classical one up to the term $(\mathbf{u . n}) \mathbf{u}_{I}$. This is an additional forcing term. Without this term, it is easy checked that one can only derive an à priori estimate when a smallness assumption on $\mathbf{u}_{I}$ is satisfied, an assumption which would restrict the problem to a laminar one. Therefore, this term seems to look coherent when the flow is turbulent at the incoming boundary. However, in the numerical simulations we have taken $\boldsymbol{\sigma}(\mathbf{u}, p, k) \cdot \mathbf{n}=\mathbf{0}$. Indeed, the 
experiments suggest that the flow is laminar far from the net. Therefore, our choices are in concordance with reality and yields a rigorous mathematical analysis.

Remark 4.2 For convenience and the sake of simplicity, we have chosen to develop the theoretical part by fixing $k=0$ at $\Gamma_{o}$. A more natural boundary condition at $\Gamma_{o}$ is $\mu_{t} \frac{\partial k}{\partial \mathbf{n}}=0$. This is the condition that we use in the numerical simulations. From the mathematical viewpoint, we then have to impose $\mu_{t} \frac{\partial k}{\partial \mathbf{n}}=-(\mathbf{u . n})^{-} k$ at $\Gamma_{o}$. Therefore the discussion in remark 4.1 above holds in this case. However, this boundary condition yields serious mathematical complications that would have been out of the scope of this paper. In subsection 5.5 we give some explanations about this case.

\subsubsection{Initial data}

The initial data are specified by

$$
\begin{array}{ll}
\forall \mathbf{x} \in \Omega, & \mathbf{u}(0, \mathbf{x})=\mathbf{u}_{0}(\mathbf{x}) \in\left(L^{2}(\Omega)\right)^{2}, \\
\forall \mathbf{x} \in \Omega, & k(0, \mathbf{x})=k_{0}(\mathbf{x}) \in L^{1}(\Omega) .
\end{array}
$$

Moreover, we shall assume that $\mathbf{u}_{0}$ satisfies the compatibility conditions

$$
\begin{aligned}
& \nabla \cdot \mathbf{u}_{0}=0 \\
& \mathbf{u}_{0} \cdot \mathbf{n}=u_{\mathrm{I}} \quad \text { on } \Gamma_{i}, \\
& \mathbf{u}_{0} \cdot \mathbf{n}=0 \quad \text { on } \Gamma_{l} .
\end{aligned}
$$

\subsection{The eddy viscosities and main terms}

\subsubsection{Eddy viscosities}

The eddy viscosity function $\nu_{t}$ is a $C^{1}$ non negative bounded function of $k$ and $\mathbf{x}$ equal to $\nu_{0}+\ell(\mathbf{x}) \sqrt{\tau+|k|}$ when $|k| \in\left[0, k_{c}\right]$ for a given $k_{c}$ and $\tau>0$ is fixed. The viscosity $\nu_{t}$ is thus given by

$$
\begin{aligned}
& \nu_{t}(k, \mathbf{x})=\nu_{0}+\ell(\mathbf{x}) \sqrt{\tau+|k|}, \quad \text { when }|k| \leq k_{c} \\
& \nu_{t}(k, \mathbf{x})=v_{2}, \quad \text { when }|k| \geq k_{c}+1
\end{aligned}
$$




$$
\left\{\begin{array}{l}
\nu_{t}(k, \mathbf{x})=\left(\frac{l(x)}{2 \sqrt{\tau+k_{c}}}+2 v_{1}-2 v_{2}\right) k^{3}+\left(\frac{l(x)\left(-3 k_{c}-2\right)}{2 \sqrt{\tau+k_{c}}}+\right. \\
\left.\left(v_{1}-v_{2}\right)\left(-6 k_{c}-3\right)\right) k^{2}+\left(\frac{l(x)\left(1+3 k_{c}^{2}+4 k_{c}\right)}{2 \sqrt{\tau+k_{c}}}+\left(v_{1}-v_{2}\right)\left(6 k_{c}^{2}+6 k_{c}\right)\right) k+ \\
v_{1}+\frac{l(x)\left(-k_{c}^{3}-2 k_{c}^{2}-k_{c}\right)}{2 \sqrt{\tau+k_{c}}}+\left(v_{1}-v_{2}\right)\left(-2 k_{c}^{3}-3 k_{c}^{2}\right) \quad \text { when } k_{c}<k<k_{c}+1
\end{array}\right.
$$

where $\tau>0, \quad k_{c}>0, \quad v_{2}>v_{1}=\nu_{0}+\ell(\mathbf{x}) \sqrt{\tau+k_{c}}$.

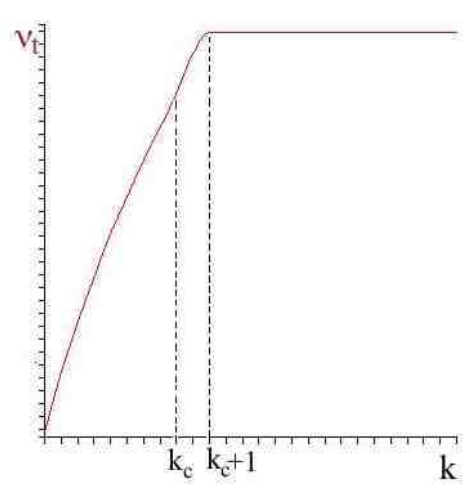

Fig. 7. Shape of $\nu_{t}$

The function $\ell(\mathbf{x})$ is a local scale of the flow. It is a non negative bounded $C^{1}$ function of $\mathbf{x}$ on $\Omega$ with

$$
\forall \mathrm{x} \in \Omega, \quad 0<\ell_{0} \leq \ell(x) \leq L_{0}<\infty .
$$

The eddy diffusivity $\mu_{t}$ is of the same form as $\nu_{t}$ and

$$
\nu_{0}+C \ell(\mathbf{x}) \sqrt{\tilde{\tau}+|k|} \text { on the range }\left[0, k_{c}\right]
$$

for $C>0$ and $\tilde{\tau}>0$ fixed coefficients.

\subsubsection{Backward term}

The backward term $\mathcal{E}(k, \mathbf{x})$ is given by the formula

$$
\mathcal{E}(k, \mathbf{x})=\frac{1}{\ell(\mathbf{x})} k \sqrt{k} .
$$

\subsubsection{Permeability}

The permeability function $K(\mathbf{x})$ is a continuous function that satisfies

$$
\forall \mathbf{x} \in \Omega, \quad 0<K_{0} \leq K(\mathbf{x}) \leq K_{1}<\infty .
$$

In the remainder, one shall set

$$
\mathcal{P}(\mathbf{u})(t, \mathbf{x})=\left(\frac{1}{\varepsilon}\left(\mathbb{I}_{G_{f} \cup G_{c}}(\mathbf{x})\right)+\frac{1}{K(\mathbf{x})} \mathbb{I}_{G_{n}}(\mathbf{x})+\varepsilon \mathbb{I}_{\Omega_{w}}\right) \mathbf{u}(t, \mathbf{x}),
$$


where $\varepsilon>0$ is fixed.

\section{$5 \quad$ Mathematical analysis}

\subsection{Main result}

We summerize the hypotheses:

$$
\begin{aligned}
& \nu_{t} \in C^{1}, \quad \forall(k, \mathbf{x}) \in \mathbb{R} \times \Omega, \quad 0<\nu_{0} \leq \nu_{t}(k, \mathbf{x}) \leq N<\infty, \\
& \mu_{t} \in C^{1}, \quad \forall(k, \mathbf{x}) \in \mathbb{R} \times \Omega, \quad 0<\mu_{0} \leq \mu_{t}(k, \mathbf{x}) \leq M<\infty, \\
& \ell \in L^{\infty}, \quad \forall \mathbf{x} \in \Omega, \quad 0<\ell_{0} \leq \ell(x) \leq L_{0}<\infty \\
& \mathcal{E}(k, \mathbf{x})=\frac{1}{\ell(\mathbf{x})} k \sqrt{|k|}, \\
& K \in C^{1}, \quad \forall \mathbf{x} \in \Omega, \quad 0<K_{0} \leq K(\mathbf{x}) \leq K_{1}<\infty . \\
& \mathbf{u}_{0} \in L^{2}(\Omega), \quad \nabla \cdot \mathbf{u}_{0}=0,\left.\quad \mathbf{u}_{0} \cdot \mathbf{n}\right|_{\Gamma_{i}}=u_{\mathrm{I}},\left.\quad \mathbf{u}_{0} \cdot \mathbf{n}\right|_{\Gamma_{l}}=0, \\
& k_{0} \in L^{1}(\Omega), \quad k_{0} \geq 0 \text { a.e } \\
& \mathbf{u}_{\mathrm{I}} \in H_{00}^{3 / 2}\left(\Gamma_{i}\right) .
\end{aligned}
$$

The problem is the following

$$
\begin{aligned}
& \partial_{t} \mathbf{u}+(\mathbf{u} \nabla) \mathbf{u}-\nabla \cdot \boldsymbol{\sigma}(\mathbf{u}, p, k)+\mathcal{P}(\mathbf{u})=\mathbf{0}, \\
& \nabla \cdot \mathbf{u}=0, \\
& \partial_{t} k+\mathbf{u} \cdot \nabla k-\nabla \cdot\left(\mu_{t}(k, \mathbf{x}) \nabla k\right)=2 \nu_{t}(k, \mathbf{x})|\varepsilon(\mathbf{u})|^{2}-\mathcal{E}(k, \mathbf{x}) . \\
& \forall \mathbf{x} \in \Omega, \quad \mathbf{u}(0, \mathbf{x})=\mathbf{u}_{0}(\mathbf{x}), \\
& \forall \mathbf{x} \in \Omega, \quad k(0, \mathbf{x})=k_{0}(\mathbf{x}), \\
& \left.\mathbf{u}\right|_{\Gamma_{i}}=\mathbf{u}_{\mathrm{I}}=\left(u_{\mathrm{I}}, 0\right),\left.\quad k\right|_{\Gamma_{i}}=0, \\
& \left.\mathbf{u}\right|_{\Gamma_{l}}=\mathbf{0},\left.\quad k\right|_{\Gamma_{l}}=0, \\
& \left.\boldsymbol{\sigma}(\mathbf{u}, p, k) \cdot \mathbf{n}\right|_{\Gamma_{o}}=-\frac{1}{2}(\mathbf{u} . \mathbf{n})^{-}\left(\mathbf{u}-\mathbf{u}_{\mathrm{I}}\right)+(\mathbf{u} \cdot \mathbf{n}) \mathbf{u}_{\mathrm{I}}, \\
& \left.k\right|_{\Gamma_{o}}=0 .
\end{aligned}
$$

Assumption (29) above has to be clarified. We shall say that a given field $\mathbf{v}$ defined on $\Gamma_{l}$ is in the space $H_{00}^{s}(s>0$ being given $)$ if and only if

$$
\int_{0}^{\alpha} \frac{|\mathbf{v}(y)|^{2}}{y^{2 s}} d y<+\infty \text { and } \int_{0}^{\alpha} \frac{|\mathbf{v}(y)|^{2}}{(\alpha-y)^{2 s}} d y<+\infty
$$

see for instance in [20], chapter $1, \S 11$ or in [8] chapter 6 . This assumption is a technical assumption telling that the field $\mathbf{v}$ goes to zero sufficiently fast at the edges. In our problem, we take $s=3 / 2$ in order to extend $u_{\mathrm{I}}$ by zero on $\Gamma_{l}$ with 
enough regularity because of the no slip condition on $\Gamma_{l}$ (see Remark 5.3 in the remainder). Therefore, as we shall see it in the following, it will be possible to lift the boundary condition then including the information in the equations with enough regularity, so that we can work with homogeneous boundary conditions. This is more appropriate to the mathematical analysis.

In numerical simulations, we took a natural uniform $u_{\mathrm{I}}$ on $\Gamma_{i}$ for the sake of simplicity. To avoid an articial boundary layer, we impose a slip condition for the velocity on $\Gamma_{l}$. However, we are not able to prove any theoretical existence result for such a problem. This is not surprising since this is one of the usual gap between mathematical theory and numerical simulations while working with Navier-Stokes equations and by-products. This is also linked to the physical discussion to know if the no slip condition at the boundaries is reasonnable or not, even if it yields a good mathematical structure.

Finally, we could also do the mathematical analysis with a uniform flow at $\Gamma_{i}$ by extending it from its value at the edges to zero on $\Gamma_{l}$ sufficiently closely to the edges while keeping a $H_{00}^{s}$ regularity type. Therefore, we would have a mathematical structure "asymptotically" close from the numerical structure. This will not change at all our mathematical analysis but just make it more heavy than it is already.

Our main result is the following.

Theorem 5.1 Assume that hypotheses [(22)...(29)] hold. Then Problem [(30)...(38)] admits a solution $(\mathbf{u}, p, k)$ on any time interval $[0, T]$ in the sense of the distributions, where

$$
\begin{aligned}
& \mathbf{u} \in L^{2}\left([0, T],\left(H^{1}(\Omega)\right)^{2}\right) \cap L^{\infty}\left([0, T], L^{2}(\Omega)\right), \\
& p \in L^{2}([0, T] \times \Omega), \\
& k \in L^{\infty}\left([0, T], L^{1}(\Omega)\right) \cap\left(\bigcap_{p<\frac{4}{3}} L^{p}\left([0, T], W^{1, p}(\Omega)\right)\right) .
\end{aligned}
$$

Remark 5.1 Uniqueness remains an open problem.

\subsection{Lifting the boundary condition}

\subsubsection{Auxiliary Stokes Problem}

In this section, we describe how to lift the boundary conditions to reduce the problem to a problem with homogeneous boundary conditions on $\Gamma_{i} \cup \Gamma_{l}$, as it is usually done in mathematical problems where Navier-Stokes Equations are involved.

Recall that $\Omega_{w}$ is the water domain and $G$ the net domain (see section 3.1). 
The incoming flow $\mathbf{u}_{\mathrm{I}}$ is prescribed at the boundary $\Gamma_{i}$. We define $\mathbf{u}_{\mathrm{I}}$ on the output boundary $\Gamma_{o}$ and still denote it by $\mathbf{u}_{\mathrm{i}}$, the field defined by

$$
\forall \mathbf{x}=(x, y) \in \Gamma_{o}, \quad \mathbf{u}_{\mathrm{I}}(x, y)=\left(u_{\mathrm{I}}(x-\beta, 0)\right) .
$$

Let us consider the Stokes problem

$$
\begin{gathered}
-\Delta \mathbf{v}_{0}+\nabla q_{0}=\mathbf{0} \quad \text { in } \Omega_{w} \\
\nabla \cdot \mathbf{v}_{0}=0 \quad \text { in } \Omega_{w}, \\
\mathbf{v}_{0}=\mathbf{g} \quad \text { on } \Gamma \cup \partial G
\end{gathered}
$$

where $\Omega_{w}$ is the water domain, $G$ the domain delimited by the net and $\mathbf{g}$ is the field defined by

$$
\begin{aligned}
& \text { on } \Gamma_{i} \cup \Gamma_{o}, \mathbf{g}=\mathbf{u}_{\mathrm{I}}, \\
& \text { on } \Gamma_{l} \cup \partial G, \mathbf{g}=\mathbf{0},
\end{aligned}
$$

Notice that the following compatibility condition is satisfied:

$$
\int_{\Gamma} \mathbf{g} \cdot \mathbf{n}=\mathbf{0}
$$

In the following, we note

$$
L_{0}^{2}(\Omega)=\left\{q \in L^{2}(\Omega) ; \int_{\Omega} q(\mathbf{x}) d \mathbf{x}=0\right\}
$$

Theorem 5.2 Assume that $u_{I} \in H_{00}^{3 / 2}\left(\Gamma_{i}\right)$ (assumption (29)). Then Problem $[(42)-$ (43)] has a unique solution $\left(\mathbf{v}_{0}, q_{0}\right) \in H^{2}\left(\Omega_{w}\right) \times\left(H^{1}\left(\Omega_{w}\right) \cap L_{0}^{2}\left(\Omega_{w}\right)\right)$.

Proof. On one hand, it is established by Corollary 5.9 in $[9]$ that $\mathbf{g} \in\left[H^{3 / 2}(\Gamma)\right]^{2}$ because $u_{\mathrm{I}} \in H_{00}^{3 / 2}\left(\Gamma_{i}\right)$. On the other hand, $\mathrm{g}$ satisfies the compatibility condition (44). Moreover, $\Omega_{w}$ is a convex polygon in dimension 2 . Therefore, applying Theorem 5.4 and Remark 5.6 in [11] $\S$ I. 5 (see also [12]), one knows the existence of a unique $\left(\mathbf{v}_{0}, q_{0}\right) \in H^{2}\left(\Omega_{w}\right) \times\left(H^{1}\left(\Omega_{w}\right) \cap L_{0}^{2}\left(\Omega_{w}\right)\right)$ solution to Problem [(42) - (43)].

Remark 5.2 Since $\mathbf{g} \in\left[H^{3 / 2}(\Gamma)\right]^{2}$, the trace on $\Gamma_{o}$ of $\varepsilon\left(\mathbf{v}_{0}\right)$ is in $\left[H^{1 / 2}\left(\Gamma_{o}\right)\right]^{4}$ as well as the trace of $q_{0}$ on $\Gamma_{o}$ is in $H^{1 / 2}\left(\Gamma_{o}\right)$. Then, because $\nu_{t}$ is a bounded function, for every $k \in L^{1}(\Omega)$,

$$
\boldsymbol{\sigma}\left(\mathbf{v}_{0}, q_{0}, k\right) \in\left[H^{1 / 2}\left(\Gamma_{o}\right)\right]^{4}
$$

From now, one still denotes by $\mathbf{v}_{0}$ the field defined on whole $\Omega$ and equal to $\mathbf{v}_{0}$ in $\Omega_{w}$, the velocity part in the solution to Problem [(42) - (43)], and equal to 0 inside $G$. Since

- $H^{2}\left(\Omega_{w}\right) \subset L^{\infty}\left(\Omega_{w}\right)$

- $\partial G$ is of class $C^{1}$, therefore one can use Proposition IX.18 in [6], 
one has

$$
\mathbf{v}_{0} \in H^{1}(\Omega) \cap L^{\infty}(\Omega)
$$

and

$$
\left\|\mathbf{v}_{0}\right\|_{H^{1}(\Omega)}+\left\|\mathbf{v}_{0}\right\|_{L^{\infty}(\Omega)} \leq C\left\|u_{\mathrm{I}}\right\|_{H_{00}^{3 / 2}\left(\Gamma_{i}\right)},
$$

where $C$ only depends on $\alpha$ and $\beta$. By extending $q_{0}$ by zero outside $\Omega_{w}$ and still denoting the expension by $q_{0}$, one has

$$
\boldsymbol{\sigma}\left(\mathbf{v}_{0}, q_{0}, k\right) \in\left[L^{2}(\Omega)\right]^{4} .
$$

Notice also that

$$
\mathcal{P}\left(\mathbf{v}_{0}\right)=\mathbf{0}
$$

Remark 5.3 The choice of the space $H_{00}^{3 / 2}\left(\Gamma_{i}\right)$ is motivated by the estimate (47). Indeed, we need that the lifting field $\mathbf{v}_{0}$ not only lies in a $H^{1}$ space type but also is bounded. Of course, as we said already, this a technical assumption and many variants can be imagined.

\subsubsection{Change of variable}

We set:

$$
\mathbf{u}=\tilde{\mathbf{u}}+\mathbf{v}_{0}, \quad p=\tilde{p}+q_{0} .
$$

It is straightforward to prove that $(\tilde{\mathbf{u}}, \tilde{p}, k)$ is governed by the following system:

$$
\begin{aligned}
& \left\{\begin{array}{l}
\partial_{t} \tilde{\mathbf{u}}+(\tilde{\mathbf{u}} \nabla) \tilde{\mathbf{u}}-\nabla \cdot \boldsymbol{\sigma}(\tilde{\mathbf{u}}, \tilde{p}, k)+\mathcal{P}(\tilde{\mathbf{u}})+ \\
(\tilde{\mathbf{u}} \nabla) \mathbf{v}_{0}+\left(\mathbf{v}_{0} \nabla\right) \tilde{\mathbf{u}}+\left(\mathbf{v}_{0} \nabla\right) \mathbf{v}_{0}-\nabla \cdot \boldsymbol{\sigma}\left(\mathbf{v}_{0}, q_{0}, k\right)=\mathbf{0},
\end{array}\right. \\
& \nabla \cdot \tilde{\mathbf{u}}=0, \\
& \left\{\begin{array}{l}
\partial_{t} k+\tilde{\mathbf{u}} \cdot \nabla k-\nabla \cdot\left(\mu_{t}(k, \mathbf{x}) \nabla k\right)=2 \nu_{t}(k, \mathbf{x})|\boldsymbol{\varepsilon}(\tilde{\mathbf{u}})|^{2}-\mathcal{E}(k, \mathbf{x})+ \\
4 \nu_{t}(k, \mathbf{x}) \boldsymbol{\varepsilon}(\tilde{\mathbf{u}}) \cdot \boldsymbol{\varepsilon}\left(\mathbf{v}_{0}\right)+2 \nu_{t}(k, \mathbf{x})\left|\boldsymbol{\varepsilon}\left(\mathbf{v}_{0}\right)\right|^{2}-\mathbf{v}_{0} \nabla k .
\end{array}\right. \\
& \left.\tilde{\mathbf{u}}\right|_{t=0}=\mathbf{u}_{0}-\mathbf{v}_{0},\left.\quad k\right|_{t=0}=k_{0}, \\
& \left.\tilde{\mathbf{u}}\right|_{\Gamma_{i} \cup \Gamma_{l}}=\mathbf{0},\left.\quad k\right|_{\Gamma_{i} \cup \Gamma_{l}}=0, \\
& \left\{\begin{array}{l}
\left.\boldsymbol{\sigma}(\tilde{\mathbf{u}}, \tilde{p}, k) \cdot \mathbf{n}\right|_{\Gamma_{o}}= \\
-\frac{1}{2}\left[\left(\tilde{\mathbf{u}}+\mathbf{v}_{0}\right) \cdot \mathbf{n}\right]^{-} \tilde{\mathbf{u}}+\left[\left(\tilde{\mathbf{u}}+\mathbf{v}_{0}\right) \cdot \mathbf{n}\right] \mathbf{v}_{0}-\left.\boldsymbol{\sigma}\left(\mathbf{v}_{0}, q_{0}, k\right)\right|_{\Gamma_{o}} \cdot \mathbf{n},
\end{array}\right. \\
& \left.k\right|_{\Gamma_{o}}=0 .
\end{aligned}
$$




\subsection{Variational formulation}

\subsubsection{Functions space}

The natural space for studying Problem $[(51)--(56)]$ is the space

$$
V=\left\{\mathbf{v} \in\left(H^{1}(\Omega)\right)^{2} ; \quad \nabla \cdot \mathbf{v}=0 ;\left.\quad \mathbf{v}\right|_{\Gamma_{i} \cup \Gamma_{l}}=\mathbf{0} .\right\}
$$

In order to use De Rham Theorem and have an Inf-Sup condition on the pressure, we must check that smooth vector fields with null divergence and equal to zero on $\Gamma_{i} \cup \Gamma_{l}$ consitutes a dense space in $V$. This is the goal of what follows.

Let $\tilde{B}=(2 \beta, \alpha), \tilde{C}=(2 \beta, 0)$ and let $\tilde{\Omega}$ be the square in $\mathbb{R}^{2}$ bounded by the points $O, A, \tilde{B}$ and $\tilde{C}$. Let $s$ be the symmetry through the axis $x=\beta$, that is $s(x, y)=(2 \beta-x, y)$.

We also denote by $\Omega^{s}$ the square bounded by the points $C, \tilde{C}, \tilde{B}$ and $B$, also defined by $\Omega^{s}=s(\Omega)$.

Let $\tilde{V}$ be the set

$$
\tilde{V}=\left\{\mathbf{v} \in\left(H^{1}(\tilde{\Omega})\right)^{2} ; \quad \nabla \cdot \mathbf{v}=0 ;\left.\quad \mathbf{v}\right|_{\partial \tilde{\Omega}}=\mathbf{0}\right\}
$$

as well as

$$
\tilde{\mathcal{V}}=\left\{\mathbf{v} \in(\mathcal{D}(\tilde{\Omega}))^{2} ; \quad \nabla \cdot \mathbf{v}=0\right\} .
$$

Being given $\mathbf{v} \in \tilde{V}$, let $\mathbf{v}_{r}$ be its restriction to the square $\Omega$. One obviously has $\mathbf{v}_{r} \in V$.

Being given $\mathbf{v} \in V$, let $\mathbf{v}^{e}$ be its extension to $\tilde{\Omega}$ defined as follows:

$$
\begin{aligned}
& \forall \mathbf{x} \in \Omega, \quad \mathbf{v}^{e}(\mathbf{x})=\mathbf{v}(\mathbf{x}), \\
& \forall \mathbf{x} \in \Omega^{s}, \mathbf{v}^{e}(\mathbf{x})=\mathbf{v}(s(\mathbf{x}))
\end{aligned}
$$

Notice that $\mathbf{v}^{e} \in \tilde{V}$ and one has

$$
\int_{\Omega^{s}}\left|\nabla \mathbf{v}^{e}\right|^{2}=2 \int_{\Omega}|\nabla \mathbf{v}|^{2}, \quad \forall p \in\left[1, \infty\left[, \quad \int_{\Omega^{s}}\left|\mathbf{v}^{e}\right|^{p}=2 \int_{\Omega}|\mathbf{v}|^{p} .\right.\right.
$$

Finally let $\mathcal{V}$ be the space made of the restrictions to $\Omega$ of fields in $\tilde{\mathcal{V}}$, which means

$$
\mathcal{V}=\left\{\mathbf{v} \in\left[C^{\infty}(\Omega)\right] ; \exists \mathbf{v} \in \tilde{\mathcal{V}} \text { s.t. } \mathbf{v}=\mathbf{v}_{r}\right\}
$$

We need the following lemma: 
Lemma 5.1 The space $\mathcal{V}$ is dense in $V$.

This technical result is proved in the Appendix. It is essential for our purpose since we are studying a variational formulation (see below) where the pressure disappears. Therefore, we need to apply the De Rham Theorem to check that the problem that we solved is the same as the one we are starting with.

\subsubsection{The variational Problem}

For the sake of the simplicity, up to now and throughout the paper we shall note $\nu_{t}(k)$ instead of $\nu_{t}(k, \mathbf{x})$. Notice firstly that $\forall\left(\mathbf{v}_{1}, \mathbf{v}_{2}\right) \in \mathcal{V}^{2}, \forall(k, q) \in \mathcal{D}(\Omega)^{2}$ one has

$$
-\int_{\Omega}\left(\nabla \cdot \boldsymbol{\sigma}\left(\mathbf{v}_{1}, q, k\right)\right) \cdot \mathbf{v}_{2}=-\int_{\Gamma_{o}}\left(\boldsymbol{\sigma}\left(\mathbf{v}_{1}, q, k\right) \cdot \mathbf{n}\right) \cdot \mathbf{v}_{2}+\int_{\Omega} 2 \nu_{t}(k) \boldsymbol{\varepsilon}\left(\mathbf{v}_{1}\right): \boldsymbol{\varepsilon}\left(\mathbf{v}_{2}\right) .
$$

The variational formulation of the problem is the following, where the pressure does not appear anymore and will be recovered using The De Rham Theorem. In the following, one denotes

$$
W=L^{2}([0, T], V) \cap L^{\infty}\left([0, T],\left(L^{2}(\Omega)\right)^{2}\right)
$$

FIND

$$
\begin{aligned}
& \tilde{\mathbf{u}} \in W, \quad \tilde{\mathbf{u}}(0, \mathbf{x})=\mathbf{u}_{0}(\mathbf{x})-\mathbf{v}_{0}(\mathbf{x}) \quad \text { a.e in } \Omega \\
& k \in L^{\infty}\left([0, T], L^{1}(\Omega)\right) \cap\left(\bigcap_{p<4 / 3} L^{p}\left([0, T], W^{1, p}(\Omega)\right)\right)
\end{aligned}
$$

WITH

$$
\partial_{t} \tilde{\mathbf{u}} \in L^{8 / 5}\left([0, T], V^{\prime}\right) \cap W^{\prime},
$$

AND SUCH THAT $\forall \mathbf{v} \in L^{2}([0, T], V)$,

$$
\begin{aligned}
& <\partial_{t} \tilde{\mathbf{u}}, \mathbf{v}>+\int_{0}^{T} \int_{\Omega}(\tilde{\mathbf{u}} \nabla) \tilde{\mathbf{u}} \cdot \mathbf{v}+\int_{0}^{T} \int_{\Omega} 2 \nu_{t}(k) \varepsilon(\tilde{\mathbf{u}}): \varepsilon(\mathbf{v})+\int_{0}^{T} \int_{\Gamma_{o}} \frac{1}{2}\left[\left(\tilde{\mathbf{u}}+\mathbf{v}_{0}\right) \cdot \mathbf{n}\right]^{-} \tilde{\mathbf{u}} \cdot \mathbf{v}- \\
& \int_{0}^{T} \int_{\Gamma_{o}}\left[\left(\tilde{\mathbf{u}}+\mathbf{v}_{0}\right) \cdot \mathbf{n}\right] \mathbf{v}_{0} \cdot \mathbf{v}+\int_{0}^{T} \int_{\Omega} \mathcal{P}(\tilde{\mathbf{u}}) \cdot \mathbf{v}+\int_{0}^{T} \int_{\Omega}\left[\left(\mathbf{v}_{0} \nabla\right)\left(\tilde{\mathbf{u}}+\mathbf{v}_{0}\right) \cdot \mathbf{v}+\right. \\
& \int_{0}^{T} \int_{\Omega} 2 \nu_{t}(k) \varepsilon\left(\mathbf{v}_{0}\right): \varepsilon(\mathbf{v})+\int_{0}^{T} \int_{\Gamma_{o}}\left(\boldsymbol{\sigma}\left(\mathbf{v}_{0}, q_{0}, k\right) \cdot \mathbf{n}\right) \cdot \mathbf{v}=0,
\end{aligned}
$$

FOR ALL $r \in \mathcal{D}^{\prime}([0, T] \times \Omega)$, WITH $r(T, \cdot)=0$,

$$
\begin{aligned}
& -\int_{0}^{T} \int_{\Omega} \partial_{t} r k+\int_{0}^{T} \int_{\Omega}\left(\left(\tilde{\mathbf{u}}+\mathbf{v}_{0}\right) \nabla\right) k \cdot r+\int_{0}^{T} \int_{\Omega} \mu_{t}(k) \nabla k: \nabla r+\int_{\Omega} k_{0}(\mathbf{x}) r(0, \mathbf{x}) d \mathbf{x}= \\
& \int_{0}^{T} \int_{\Omega}\left[2 \nu_{t}(k)|\varepsilon(\tilde{\mathbf{u}})|^{2}-\mathcal{E}(k)+\nu_{t}(k)\left(4 \varepsilon(\tilde{\mathbf{u}}) \varepsilon\left(\mathbf{v}_{0}\right)+2\left|\varepsilon\left(\mathbf{v}_{0}\right)\right|^{2}\right)\right] r
\end{aligned}
$$




\subsubsection{Consistency of the variational formulation and à priori estimates}

The variational formulation for the $k$-equation is the classical one, as in [16], [17], [18] and [15]. The variational formulation for the velocity is also classical up to the boundary terms. Each boundary term where $\mathbf{v}_{0}$ is involved is nice since $\mathbf{v}_{0}$ does not depend uppon the time and is equal to $\mathbf{u}_{\mathbf{I}}$ on $\Gamma_{o}$ which is in particular in $L^{\infty}\left(\Gamma_{i}\right)$. Nevertheless the term

$$
\int_{0}^{T} \int_{\Gamma_{o}}[\tilde{\mathbf{u}} \cdot \mathbf{n}]^{-} \tilde{\mathbf{u}} \cdot \mathbf{v}
$$

is fearsome. For the sake of simplicity and as far as no confusion occurs, we still denote by $\mathbf{v}$ the trace of $\mathbf{v}$ for any $\mathbf{v} \in W$. Moreover, one defines the $W$ norm by

$$
\|\mathbf{v}\|_{W}=\|\mathbf{v}\|_{L^{2}([0, T], V)}+\|\mathbf{v}\|_{L^{\infty}\left([0, T],\left(L^{2}(\Omega)^{2}\right)\right.} .
$$

The following results are basic estimates we need to prove our main result. Interested readers may find the proofs in the Appendix.

The following lemma guarantees the consistency of the variational formulation above.

Lemma 5.2 Let $(\tilde{\mathbf{u}}, \mathbf{v}) \in W \times W$. Then

$$
\int_{0}^{T} \int_{\Gamma_{o}}[\tilde{\mathbf{u}} . \mathbf{n}]^{-} \tilde{\mathbf{u}} \cdot \mathbf{v} \leq C\|\tilde{\mathbf{u}}\|_{W}^{2}\|\mathbf{v}\|_{W}
$$

where $C$ is a constant that only depends on $\alpha$ and $\beta$. Moreover, there also exists a constant $\tilde{C}$ such that

$$
\forall \tilde{\mathbf{v}} \in L^{8 / 3}([0, T], V), \quad \int_{0}^{T} \int_{\Gamma_{o}}[\tilde{\mathbf{u}} . \mathbf{n}]^{-} \tilde{\mathbf{u}} \cdot \mathbf{v} \leq C\|\tilde{\mathbf{u}}\|_{W}^{2}\|\tilde{\mathbf{v}}\|_{L^{8 / 3}([0, T], V)}
$$

Proposition 5.1 There exists a constant $C_{1}=C_{1}\left(\mathbf{u}_{0}, u_{I}, \nu, \alpha, \beta\right)$ and for each $p<$ $4 / 3$ a constant $C_{2}=C_{2}\left(\mathbf{u}_{0}, u_{I}, \nu, \mu, p, \alpha, \beta\right)$ such that for any smooth solution $(\tilde{\mathbf{u}}, k)$ to the variational problem $[(67),(66)]$ one has

$$
\begin{aligned}
& \|\tilde{\mathbf{u}}\|_{L^{2}([0, T], V)}+\|\tilde{\mathbf{u}}\|_{L^{\infty}\left([0, T], L^{2}(\Omega)\right)} \leq C_{1}, \\
& \|k\|_{L^{p}\left([0, T], W^{1, p}(\Omega)\right)} \leq C_{2} .
\end{aligned}
$$

\subsection{End of the proof of the main Theorem}

The proof now is the same as in [16], [17], [18] and [15], up to the additional terms due to the extra boundary conditions for the velocity. We construct a sequence of smooth approximated solution $\left(\tilde{\mathbf{u}}_{n}, k_{n}\right)_{n \in \mathbb{N}}$ (for instance by troncating the l.h.s of the $k$-equation and using the Galerkin method). The trick is to prove the weak convergence in $L^{2}([0, T], V)$ of the sequence $\left(\tilde{\mathbf{u}}_{n}\right)_{n \in \mathbb{N}}$ (up to a subsequence) to $\tilde{\mathbf{u}} \in W$ which satisfies the formulation (66), and in particular, that can be taken as a test function in (66). Once this task is finished, the rest is classical and works as in [16], 
[17], [18] and [15] since we already have obtained all the required à priori estimates.

Let $\mathbf{u} \in W$. One has, after a part integration on the convective term,

$$
\begin{aligned}
& <\partial_{t} \tilde{\mathbf{u}}_{n}, \mathbf{v}>-\int_{0}^{T} \int_{\Omega} \tilde{\mathbf{u}}_{n} \otimes \tilde{\mathbf{u}}_{n} \nabla \mathbf{v}+\int_{0}^{T} \int_{\Gamma_{o}}\left(\tilde{\mathbf{u}}_{n} \cdot \mathbf{n}\right) \tilde{\mathbf{u}}_{n} \cdot \mathbf{v}+\int_{0}^{T} \int_{\Omega} 2 \nu_{t}\left(k_{n}\right) \varepsilon\left(\tilde{\mathbf{u}}_{n}\right): \varepsilon(\mathbf{v}) \\
& +\int_{0}^{T} \int_{\Gamma_{o}} \frac{1}{2}\left[\left(\tilde{\mathbf{u}}_{n}+\mathbf{v}_{0}\right) \cdot \mathbf{n}\right]^{-} \tilde{\mathbf{u}}_{n} \cdot \mathbf{v}-\int_{0}^{T} \int_{\Gamma_{o}}\left[\left(\tilde{\mathbf{u}}_{n}+\mathbf{v}_{0}\right) \cdot \mathbf{n}\right] \mathbf{v}_{0} \cdot \mathbf{v}+ \\
& \int_{0}^{T} \int_{\Omega} \mathcal{P}\left(\tilde{\mathbf{u}}_{n}\right) \cdot \mathbf{v}+\int_{0}^{T} \int_{\Omega}\left[\left(\mathbf{v}_{0} \nabla\right)\left(\tilde{\mathbf{u}}_{n}+\mathbf{v}_{0}\right) \cdot \mathbf{v}+\int_{0}^{T} \int_{\Omega} 2 \nu_{t}(k) \varepsilon\left(\mathbf{v}_{0}\right): \varepsilon(\mathbf{v})+\right. \\
& \int_{0}^{T} \int_{\Gamma_{o}}\left(\boldsymbol{\sigma}\left(\mathbf{v}_{0}, q_{0}, k\right) \cdot \mathbf{n}\right) \cdot \mathbf{v}=0,
\end{aligned}
$$

By using (70), one knows that the sequence $\left(\mathbf{u}_{n}\right)_{n \in \mathbb{N}}$ is bounded in $W$ and one may extract a subsequence (still denoted by the same) that weakly converges in $L^{2}([0, T], V)$ and in $L^{\infty}\left([0, T], L^{2}\right)$ to some $\tilde{\mathbf{u}} \in W$. One needs compactness, and for it we shall use the Aubin-Lions Lemma. Of course, all the terms involved in (66) satisfied by each $\tilde{\mathbf{u}}_{n}$ are nice except the terms

$$
\int_{0}^{T} \int_{\Gamma_{o}}\left(\tilde{\mathbf{u}}_{n} \cdot \mathbf{n}\right) \tilde{\mathbf{u}}_{n} \cdot \mathbf{v} \text { and } \int_{0}^{T} \int_{\Gamma_{o}}\left[\tilde{\mathbf{u}}_{n} \cdot \mathbf{n}\right]^{-} \tilde{\mathbf{u}}_{n} \cdot \mathbf{v}
$$

which are the worse terms and which constitutes the only new difficulty in this problem compared with previous works already quoted. Thanks to inequality (69) combined with (70), the applications

$$
\mathbf{v} \longrightarrow \int_{0}^{T} \int_{\Gamma_{o}}\left(\tilde{\mathbf{u}}_{n} \cdot \mathbf{n}\right) \tilde{\mathbf{u}}_{n} \cdot \mathbf{v}, \quad \mathbf{v} \longrightarrow \int_{0}^{T} \int_{\Gamma_{o}}\left[\tilde{\mathbf{u}}_{n} \cdot \mathbf{n}\right]^{-} \tilde{\mathbf{u}}_{n} \cdot \mathbf{v}
$$

are bounded in the space $L^{8 / 5}\left([0, T], V^{\prime}\right)((70)$ holds for the second one, the proof is the same for the first one). Since we are working in a $2 \mathrm{D}$ case, and thanks to the regularity of $\mathbf{v}_{0}$, all the other terms are bounded in $L^{2}\left([0, T], V^{\prime}\right)$. Therefore, the sequence $\left(\partial_{t} \tilde{\mathbf{u}}_{n}\right)_{n \in \mathbf{N}}$ is bounded in $L^{8 / 5}\left([0, T], V^{\prime}\right)$ as well as in $W^{\prime}$. Applying the Aubin-Lions Lemma, one concludes that the sequence $\left(\tilde{\mathbf{u}}_{n}\right)_{n \in \mathbb{N}}$ is compact in $L^{8 / 5}\left([0, T],\left(L^{2}(\Omega)\right)^{2}\right)$. Hence we are back to the usual situation concerning compactness in this type of problem. We bypass the details. We still denote by $\left(\tilde{\mathbf{u}}_{n}\right)_{n \in \mathbb{N}}$ the subsequence which converges to $\tilde{\mathbf{u}}$ almost everywhere in $\Omega$ and strongly in $L^{4}\left([0, T],\left(L^{4}(\Omega)\right)^{2}\right)$ (we are in the $2 \mathrm{D}$ case).

One has analogous compactness properties for the sequence $\left(k_{n}\right)_{n \in \mathbf{N}}$ which converges weakly in each $L^{p}\left([0, T], W_{0}^{1, p}(\Omega)\right.$ ) (up to a subsequence and $p<5 / 4$ ) to some $k$ in the space $\cap_{p<5 / 4} L^{p}\left([0, T], W_{0}^{1, p}(\Omega)\right)$, almost everywhere in $\Omega$ and stronly in $L^{q}([0, T] \times \Omega)$ for some $q>1$.

Passing to the limit in all the terms in (72) is a classical game and follows proofs done already in previous papers (we are in the $2 \mathrm{D}$ case), except concerning the terms 
(73). We show how to pass to the limit in the first one, the second one being treated by the same reasoning. Notice that one has

$$
H^{1} \subset H^{3 / 4} \subset V^{\prime},
$$

the injections being dense and compact. Hence, the sequence $\left(\tilde{\mathbf{u}}_{n}\right)_{n \in N}$ is compact in the space $L^{8 / 3}\left([0, T],\left(H^{3 / 4}(\Omega)\right)^{2}\right)$. By uniqueness of the limit, it converges to $\tilde{\mathbf{u}}$ in this space. Following the chain rule of the proof of Lemma 5.2, one deduces that $\left(\tilde{\mathbf{u}}_{n} . \mathbf{n}\right)_{n \in N}$ converges strongly to $\tilde{\mathbf{u}} . \mathbf{n}$ in $L^{4}\left([0, T], L^{2}\left(\Gamma_{0}\right)\right)$ while $\left(\tilde{\mathbf{u}}_{n}\right)_{n \in \mathbb{N}}$ converges strongly to $\tilde{\mathbf{u}}$ in $L^{8 / 3}\left([0, T],\left(L^{4}\left(\Gamma_{0}\right)\right)^{2}\right.$. Therefore,

$$
\lim _{n \rightarrow \infty} \int_{0}^{T} \int_{\Gamma_{o}}\left(\tilde{\mathbf{u}}_{n} \cdot \mathbf{n}\right) \tilde{\mathbf{u}}_{n} \cdot \mathbf{u}=\int_{0}^{T} \int_{\Gamma_{o}}(\tilde{\mathbf{u}} \cdot \mathbf{n}) \tilde{\mathbf{u}} \cdot \mathbf{u} .
$$

The rest of the proof is now classical.

\subsection{Neumann Boundary Condition Type for the TKE}

We are now working in the case where $k$ does satisfy on $\Gamma_{o}$

$$
\mu_{t} \frac{\partial k}{\partial \mathbf{n}}=-(\mathbf{u . n})^{-} k
$$

instead of $k=0$. Because this case yields serious mathematical complications, we shall not give a complete proof of the existence result. We shall limit ourself to locating the difficulties, giving the main à priori estimate and to indicating the direction to take. Details will be written in a forthcoming paper.

\subsubsection{Variational Formulation}

When $k$ satisfies (74) at $\Gamma_{o}$ instead of $k=0$, the variational formulation for the k-equation becomes: for all $r \in C^{\infty}([0, T] \times \Omega)$, with $\left.r\right|_{\Gamma_{i} \cup \Gamma_{l}}=0$ and $r(T, \cdot)=0$,

$$
\begin{aligned}
& -\int_{0}^{T} \int_{\Omega} \partial_{t} r k+\int_{0}^{T} \int_{\Omega}\left(\left(\tilde{\mathbf{u}}+\mathbf{v}_{0}\right) \nabla\right) k \cdot r+\int_{0}^{T} \int_{\Omega} \mu_{t}(k) \nabla k: \nabla r+ \\
& \int_{0}^{T} \int_{\Gamma_{o}}\left(\left(\tilde{\mathbf{u}}+\mathbf{v}_{0}\right) \cdot \mathbf{n}\right)^{-} k r+\int_{\Omega} k_{0}(\mathbf{x}) r(0, \mathbf{x}) d \mathbf{x}= \\
& \int_{0}^{T} \int_{\Omega}\left[2 \nu_{t}(k)|\varepsilon(\tilde{\mathbf{u}})|^{2}-\mathcal{E}(k)+\nu_{t}(k)\left(4 \varepsilon(\tilde{\mathbf{u}}) \varepsilon\left(\mathbf{v}_{0}\right)+2\left|\varepsilon\left(\mathbf{v}_{0}\right)\right|^{2}\right)\right] r
\end{aligned}
$$

The source of difficulty is the additional term

$$
I_{k}=\int_{0}^{T} \int_{\Gamma_{o}}\left(\left(\tilde{\mathbf{u}}+\mathbf{v}_{0}\right) \cdot \mathbf{n}\right)^{-} k r
$$




\subsection{2 À priori estimate}

One starts first with the à priori estimate. We show that in the following, there is a situation where the Boccardo-Gallouët result [4] can be applied.

Let $g$ be any non decreasing non negative piecewise $C^{1}$ bounded function defined on $\mathbb{R}^{+}, G(k)=\int_{0}^{k} g\left(k^{\prime}\right) d k^{\prime}$. Notice that $G$ is non negative and thanks to the monotonicity of $g$, one has

$$
\forall k \in \mathbb{R}^{+}, \quad 0 \leq k g(k)-G(k)
$$

Therefore, by choosing $g(k)$ as test function in (67), with $\mathbf{u}=\tilde{\mathbf{u}}+\mathbf{v}_{0}$, one has

$$
\begin{aligned}
& \frac{d}{d t} G(k)+\left.\int_{\Omega} \mu_{t}(k)\left|g^{\prime}(k)\right| \nabla k\right|^{2}+\int_{\Gamma_{o}}(\mathbf{u . n})^{+} G(k)+\int_{\Gamma_{o}}(\mathbf{u . n})^{-}(k g(k)-G(k))= \\
& \int_{\Omega} g(k)\left[2 \nu_{t}(k)|\boldsymbol{\varepsilon}(\mathbf{u})|^{2}-\mathcal{E}(k)\right]
\end{aligned}
$$

Since $g$ is non negative, combining (46), (117) and (76) one has

$$
\frac{d}{d t} G(k)+\left.\int_{\Omega} \mu_{t}(k)\left|g^{\prime}(k)\right| \nabla k\right|^{2} \leq \tilde{\widetilde{C}}\|g\|_{\infty}, \quad \tilde{\tilde{C}}=\tilde{\widetilde{C}}\left(\mathbf{u}_{\mathrm{I}}, N, \nu_{0}, \alpha, \beta, T\right)
$$

Therefore one can deduce that the results in [4] apply. Therefore the estimate (71) still holds in this case.

\subsubsection{Consistency of the variational formulation}

As said already, the difficulty is due to the term $I_{k}$. From the proof of Lemma 5.2 (see in Appendix), $\left(\left(\tilde{\mathbf{u}}+\mathbf{v}_{0}\right) \cdot \mathbf{n}\right)^{-} \in L^{4}\left([0, T], L^{2}\left(\Gamma_{0}\right)\right)$. On the other hand, by combining the trace therorem with the Sobolev Theorem, it easily checked that $k \in \cap_{p<4 / 3} L^{p}\left([0, T], L^{\frac{p}{p-2}}\left(\Gamma_{0}\right)\right)$. Here the critical case is the space $L^{4 / 3}\left([0, T], L^{2}\left(\Gamma_{0}\right)\right)$, which is not achieved. Therefore, it is not guarantied that the integral $I_{k}$ is defined.

The way to go round this difficulty is to renormalize the equation for $k$, as in [17] chapter 5 and also in [23]. Roughly speaking, one does not take a test function $r$ in the equation, but $r \psi(k)$ for functions $\psi$ having compact support. Then $I_{k}$ becomes

$$
I_{k, \psi}=\int_{0}^{T} \int_{\Gamma_{o}}\left(\left(\tilde{\mathbf{u}}+\mathbf{v}_{0}\right) \cdot \mathbf{n}\right)^{-} k \psi(k) r
$$

which is defined since $k \psi(k)$ is bounded. Of course, when doing this, new terms appear in the variational formulation. This is now out of the scope of the present paper and will be the subject of a next paper. 


\section{Numerical simulations}

Simulations have been performed using the free software Freefem ++ (see [13]). It allows computations of $2 \mathrm{D}$ and axisymmetric fluid dynamics by the means of the finite elements method (FEM).

Remember that the net is modeled as a porous membrane and enclosed in a fictive cylinder. Assume that flow is also axisymmetric. Recall this is a strong hypothesis but reasonable in the case of the study of the mean velocity around a rigid net. Then the problem reduces to a 2D one. The geometry shown on Fig. 8 and drawn in Freefem ++ has an outer net profile and a catch profile in agreement with the model of Boulogne-Sur-Mer. The inner net profile is defined by the minima of the z component of the velocity located on the LDV profiles (see table 1). To take into account the difference of permeability of the net (mainly due to the variations in the mesh opening), the domain $G_{n}$ has been decomposed in 3 sub-domains: $G_{n}^{1}, G_{n}^{2}, G_{n}^{3}$.

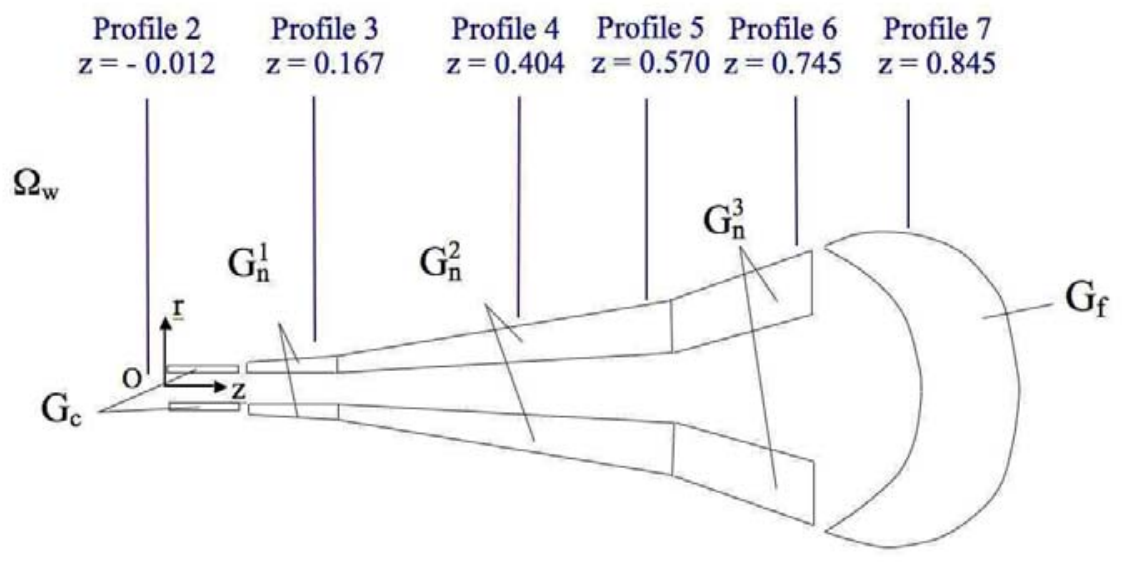

Fig. 8. Geometry of the net

Let us work in cylindrical coordinates, the $\mathrm{z}$ axis being the revolution axis of the membrane:

$$
\left\{\begin{array}{l}
x=r \cos \theta \\
y=r \sin \theta \\
z=z .
\end{array}\right.
$$

Let $\Omega=\left\{(r, z, \theta), r \in\left[r_{\min }, r_{\max }\right], z \in\left[z_{\min }, z_{\max }\right], \theta \in[0, \pi]\right\}$. Let $\mathbf{u}=\left(u_{r}, u_{\theta}, u_{z}\right)$ denote the mean velocity unknown in cylindrical coordinates.

Assuming a planar flow, then $u_{\theta}=0$ and thanks to the axisymmetric hypothesis, derivatives with respect to the variable $\theta$ are zero. 
At a fixed value of $\theta$, we work on a $2 \mathrm{D}$ domain:

$$
\Omega_{r, z}=\left\{(r, z), r \in\left[r_{\min }, r_{\max }\right], z \in\left[z_{\min }, z_{\max }\right]\right\}=\Omega_{w} \cup G_{c} \cup G_{n} \cup G_{f}
$$

Notice we keep the notations: $\Omega_{w}$ for the fluid domain, $G_{c}$ for the ring that maintains the model inside the tank, $G_{n}$ for the membrane (net) domain and $G_{f}$ for the catch domain.

In the following, the operators (gradient, divergence,...) are considered in cylindrical coordinates.

The solid part has a very small permeability, denoted $K_{s}(r, z) \ll 1$, leading to force the velocity to be zero in that part (then forcing a no slip boundary condition).

The porous part has a permeability chosen here to be constant by subdomains $G_{i}^{n}$, denoted $K_{G_{n}^{i}}, i=1,2,3$. The fluid domain has an infinite permeability so that the penalization term vanishes in that part, denoted $K_{f}(r, z) \gg 1$.

The coupled problem (30) - (38) is implemented under the following variational form.

\subsection{Weak formulation}

At first, let us assume that there is no reflexion at the outer boundary and consider the boundary conditions $(37)-(74)$ reduced to:

$$
\begin{aligned}
& \left.\boldsymbol{\sigma}(\mathbf{u}, p, k) \cdot \mathbf{n}\right|_{\Gamma_{o}}=0, \\
& \left.\frac{\partial k}{\partial \mathbf{n}}\right|_{\Gamma_{o}}=0 .
\end{aligned}
$$

Moreover, let us replace the no slip boundary condition for the velocity (see equation 36) on $\Gamma_{l}$ by slip boundary condition and the homogeneous Dirichlet condition for $k$ on $\Gamma_{l}$ by a non homogeneous one:

$$
\frac{\partial u_{z}}{\partial r}=0, \quad u_{r}=0, \quad k=k_{0} \quad \operatorname{sur} \Gamma_{l}
$$

Denote $\mathcal{V}\left(\Omega_{r, z}\right)$ and $\mathcal{Q}\left(\Omega_{r, z}\right)$ the space defined as:

$$
\mathcal{V}\left(\Omega_{r, z}\right)=\left\{\mathbf{v} \in\left(H^{1}\left(\Omega_{r, z}\right)\right)^{2}, v_{z}=0 \text { sur } \Gamma_{i}, v_{r}=0 \text { sur } \Gamma_{i} \cup \Gamma_{l}\right\},
$$




$$
\mathcal{Q}\left(\Omega_{r, z}\right)=\left\{q \in L^{2}\left(\Omega_{r, z}\right)\right\} .
$$

and

$$
\mathcal{W}\left(\Omega_{r, z}\right)=\left\{w \in L^{2}\left(\Omega_{r, z}\right), w=0 \operatorname{sur} \Gamma_{i} \cup \Gamma_{l}\right\} .
$$

A weak formulation of the coupled problem (30) - (35), with the boundary conditions (80) - (81) - (82) yields:

$$
\left\{\begin{array}{l}
\text { Find }\left(\mathbf{u}=\left(u_{r}, u_{z}\right), p, k\right) \in \mathcal{V}\left(\Omega_{r, z}\right) \times \mathcal{Q}\left(\Omega_{r, z}\right) \times \mathcal{W}\left(\Omega_{r, z}\right) \text { such that: } \\
\int_{\Omega_{r, z}} \frac{\partial \mathbf{u}}{\partial t} \mathbf{v}|r| \pi d r d z+\int_{\Omega_{r, z}}(\mathbf{u} \nabla) \mathbf{u v}|r| \pi d r d z-\int_{\Omega_{r, z}} p \nabla \cdot \mathbf{v}|r| \pi d r d z \\
+\frac{1}{2} \int_{\Omega_{r, z}}\left(\nu_{0}+\nu_{t}\right)\left(\nabla \mathbf{u}+(\nabla \mathbf{u})^{t}\right):\left(\nabla \mathbf{v}+(\nabla \mathbf{v})^{t}\right)|r| \pi d r d z \\
+\int_{\Omega_{r, z}} \mathcal{P}(\mathbf{u})(t,(r, z)) \mathbf{v}|r| \pi d r d z \\
-\int_{\Omega_{r, z}} \nabla \cdot \mathbf{u} q|r| \pi d r d z=0, \forall \mathbf{v} \in \mathcal{V}\left(\Omega_{r, z}\right), \forall q \in \mathcal{Q}\left(\Omega_{r, z}\right) ; \\
\int_{\Omega_{r, z}} \frac{\partial k}{\partial t} w|r| \pi d r d z+\int_{\Omega_{r, z}}(\mathbf{u} \nabla) k w|r| \pi d r d z+\int_{\Omega_{r, z}} \tilde{\nu}_{t}(\nabla k: \nabla w)|r| \pi d r d z \\
-\int_{\Omega_{r, z}} \frac{\nu_{t}}{2}\left|\nabla \mathbf{u}+(\nabla \mathbf{u})^{t}\right|^{2} w|r| \pi d r d z+\int_{\Omega_{r, z}} \frac{C_{3}}{\ell(\mathbf{x})} k^{\frac{3}{2}} w|r| \pi d r d z=0, \\
\forall w \in \mathcal{W}\left(\Omega_{r, z}\right) .
\end{array}\right.
$$

with

$$
\begin{aligned}
\mathcal{P}(\mathbf{u})(t,(r, z))= & \left(\frac{1}{K_{s}(r, z)}\left(\mathbb{I}_{G_{f} \cup G_{c}}(r, z)\right)+\sum_{i=1}^{3} \frac{1}{K_{G_{n}^{i}}(r, z)} \mathbb{I}_{G_{n}^{i}}(r, z)\right. \\
& \left.+\frac{1}{K_{f}(r, z)} \mathbb{I}_{\Omega_{w}}(r, z)\right) \mathbf{u}(t,(r, z))
\end{aligned}
$$




\subsection{Finite elements discretization}

Using the mesh generator of Freefem ++ , one builds an unstructured mesh $\mathcal{T}_{h}$ of the domain $\left\{(r, z), r \in\left[r_{\min }, r_{\max }\right], z \in\left[z_{\min }, z_{\max }\right]\right\}$ :

$$
\mathcal{T}_{h}=\cup_{i=1, N} K_{i}
$$

Here, $K_{i}$ are triangle elements. An example of such a mesh, built from the profiles of the different regions is shown on Fig. 9. Recall that the entire domain is meshed even inside the catch and collar regions because equations are set in the entire domain by the means of the permeability of the different media. Remark we did not make the calculations in half the domain given on figure 9 because we wanted to check the axisymmetry of the results with the use of an unstructured mesh and a parameter, $\ell(\mathbf{x})$, that depends on the local mesh side.

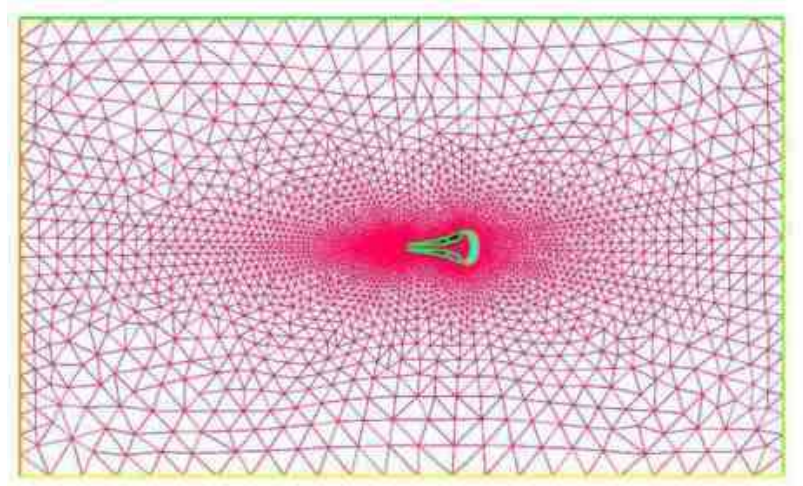

Fig. 9. Unstructured mesh of the domain $\Omega$ (10978 vertices - 21862 triangles)

Mesh refinements are located near the region $G$, since it is the region where most of the turbulence occurs.

The space discretization of the problem is based on the finite elements method. The velocity and pressure unknowns are approximated using P2/P1 finite elements.

The associated discrete finite element spaces are the following:

$$
\begin{aligned}
\mathcal{V}_{h}\left(\Omega_{r, z}\right)= & \left\{\mathbf{v}_{h}=\left(v_{r}, v_{z}\right) \in\left(\mathcal{C}^{0}\left(\Omega_{r, z}\right)\right)^{2}, \forall K_{i} \in \mathcal{T}_{h},\left.\mathbf{v}_{\mathbf{h}}\right|_{K_{i}} \in P^{2}\left(K_{i}\right),\left.\mathbf{v}_{h}\right|_{\Gamma_{i} \cap \partial K_{i}}=0\right. \\
& \text { et } \left.\left.v_{r}\right|_{\Gamma_{l} \cap \partial K_{i}}=0\right\},
\end{aligned}
$$

$$
\mathcal{Q}_{h}\left(\Omega_{r, z}\right)=\left\{q_{h} \in \mathcal{C}^{0}\left(\Omega_{r, z}\right), \forall K_{i} \in \mathcal{T}_{h}, q_{h} \in P^{1}\left(K_{i}\right)\right\}
$$


The turbulent kinetic energy $k$ is approximated by P2 finite elements.

The associated discrete finite element space is:

$$
\begin{gathered}
\mathcal{W}_{h}\left(\Omega_{r, z}\right)=\left\{w_{h} \in \mathcal{C}^{0}\left(\Omega_{r, z}\right), \forall K_{i} \in \mathcal{T}_{h}, w_{h} \in P^{2}\left(K_{i}\right),\right. \\
\left.\left.w_{h}\right|_{\Gamma_{l} \cap \partial K_{i}}=0,\left.w_{h}\right|_{\Gamma_{i} \cap \partial K_{i}}=0\right\} .
\end{gathered}
$$

The discrete weak formulation of the problem $(30)-(35),(80)-(81)-(82)$ is the following:

$$
\left\{\begin{array}{l}
\text { Finding }\left(\mathbf{u}_{h}, p_{h}, k_{h}\right) \in \mathcal{V}_{h}\left(\Omega_{r, z}\right) \times \mathcal{Q}_{h}\left(\Omega_{r, z}\right) \times \mathcal{W}_{h}\left(\Omega_{r, z}\right) \text { such that: } \\
\int_{\Omega_{r, z}} \frac{\partial \mathbf{u}_{h}}{\partial t} \mathbf{v}_{h}|r| \pi d r d z+\int_{\Omega_{r, z}}\left(\mathbf{u}_{h} \nabla\right) \mathbf{u}_{h} \mathbf{v}_{h}|r| \pi d r d z-\int_{\Omega_{r, z}} p_{h} \nabla \cdot \mathbf{v}_{h}|r| \pi d r d z \\
+\frac{1}{2} \int_{\Omega_{r, z}}\left(\nu_{0}+\nu_{t}\right)\left(\nabla \mathbf{u}_{h}+\left(\nabla \mathbf{u}_{h}\right)^{t}\right):\left(\nabla \mathbf{v}_{h}+\left(\nabla \mathbf{v}_{h}\right)^{t}\right)|r| \pi d r d z \\
+\int_{\Omega_{r, z}} \mathcal{P}\left(\mathbf{u}_{h}\right)(t, \mathbf{x}) \mathbf{v}|r| \pi d r d z \\
-\int_{\Omega_{r, z}} \nabla \cdot \mathbf{u} q|r| \pi d r d z=0, \forall \mathbf{v}_{h} \in \mathcal{V}_{h}\left(\Omega_{r, z}\right), \forall q_{h} \in \mathcal{Q}_{h}\left(\Omega_{r, z}\right) \\
\int_{\Omega_{r, z}} \frac{\partial k_{h}}{\partial t} w_{h}|r| \pi d r d z+\int_{\Omega_{r, z}}\left(\mathbf{u}_{h} \nabla\right) k_{h} w_{h}|r| \pi d r d z \\
+\int_{\Omega_{r, z}} \frac{\tilde{\nu}_{t}\left(\nabla k_{h}: \nabla w_{h}\right)|r| \pi d r d z-\int_{\Omega_{r, z}} \frac{\nu_{t}}{2}\left|\nabla \mathbf{u}_{h}+\left(\nabla \mathbf{u}_{h}\right)^{t}\right|^{2} w_{h}|r| \pi d r d z}{+\int_{\Omega_{r, z}} \frac{C_{3}}{\ell(\mathbf{x})} k_{h}^{\frac{3}{2}} w_{h}|r| \pi d r d z=0, \forall w_{h} \in \mathcal{W}_{h}\left(\Omega_{r, z}\right) .}
\end{array}\right.
$$

\subsection{Time discretization}

Denote $\delta t$ the time step. Let $\mathbf{u}_{h}^{m}, P_{h}^{m}$ and $k_{h}^{m}$ be the time approximates of the mean velocity, the modified pressure and the turbulent kinetic energy respectively, at the 
time $t^{m}=m \delta t$.

The convective terms in the problems are approximated using a characteristic Galerkin method [22], [13].

Consider a convective term like $(\mathbf{u} \nabla) \mathbf{b}$.

A Taylor expansion of the derivative

$$
\frac{D \mathbf{b}}{D t}=\frac{\partial \mathbf{b}}{\partial t}+(\mathbf{u} \nabla) \mathbf{b}
$$

yields the approximation

$$
\frac{D \mathbf{b}}{D t} \cong \frac{\mathbf{b}^{m+1}-\left(\mathbf{b}^{m}\left(x-\mathbf{u}^{m}(x) \delta t\right)\right.}{\delta t}
$$

Let $X(x, t ; s)$ be the solution of the problem:

$$
\left\{\begin{array}{l}
\frac{d X}{d s}=\mathbf{u}(X, s) \\
\left.X\right|_{s=t}=x .
\end{array}\right.
$$

$X(x, t ; s)$ is the position at time $s$ of the particule situated at position $x$ at time $t$.

Then:

$$
\frac{D \mathbf{b}}{D t} \cong \frac{\mathbf{b}^{m+1}-\mathbf{b}^{m} o X^{m}}{\delta t}
$$

where $X^{m}(x)=X\left(x, t^{n+1} ; t^{n}\right)$.

Following [22], an implicit scheme (see equation (96)) is chosen for the Navier-Stokes problem with eddy viscosity and a half-implicit one (see equation (97)) for the turbulent closure equation. 


$$
\left\{\begin{array}{l}
\text { For all } m=0, \ldots, \frac{T}{\delta t}, \\
\text { find }\left(\mathbf{u}_{h}^{m+1}, p_{h}^{m+1}, k_{h}^{m+1}\right) \in \mathcal{V}_{h}\left(\Omega_{r, z}\right) \times \mathcal{Q}_{h}\left(\Omega_{r, z}\right) \times \mathcal{W}_{h}\left(\Omega_{r, z}\right) \text { such as: } \\
\frac{1}{\delta t} \int_{\mathcal{T}_{h}}\left(\mathbf{u}_{h}^{m+1}-\mathbf{u}_{h}^{m} o X_{h}^{m}\right) \mathbf{v}_{h}|r| \pi d r d z-\int_{\mathcal{T}_{h}} p_{h}^{m+1} \nabla \cdot \mathbf{v}_{h}|r| \pi d r d z \\
+\frac{1}{2} \int_{\mathcal{T}_{h}}\left(\nu_{0}+C_{1} \ell(\mathbf{x}) \sqrt{k_{h}^{m}}\right)\left(\nabla \mathbf{u}_{h}^{m+1}+\left(\nabla \mathbf{u}_{h}^{m+1}\right)^{t}\right):\left(\nabla \mathbf{v}_{h}+\left(\nabla \mathbf{v}_{h}\right)^{t}\right)|r| \pi d r d z \\
+\int_{\mathcal{T}_{h}} \mathcal{P}\left(\mathbf{u}_{h}^{m+1}\right) \mathbf{v}_{\mathbf{h}}|r| \pi d r d z-\int_{\mathcal{T}_{h}} \nabla \cdot \mathbf{u}_{h}^{m+1} q_{h}|r| \pi d r d z \\
-\int_{\mathcal{T}_{h}} p_{h}^{m+1} q_{h} \alpha_{0}|r| \pi d r d z=0, \forall \mathbf{v}_{h} \in \mathcal{V}_{h}\left(\Omega_{r, z}\right), \forall q_{h} \in \mathcal{Q}_{h}\left(\Omega_{r, z}\right) ;
\end{array}\right.
$$

with $\alpha_{0}=10^{-7}$,

$$
\left\{\begin{array}{l}
\frac{1}{\delta t} \int_{\mathcal{T}_{h}}\left(k_{h}^{m+1}-k_{h}^{m} O X_{h}^{m}\right) w_{h}|r| \pi d r d z \\
\int_{\mathcal{T}_{h}}\left(C_{2} \ell(\mathbf{x}) \sqrt{k^{m}}\right)\left(\nabla k^{m+1}: \nabla w_{h}\right)|r| \pi d r d z \\
+\frac{1}{2} \int_{\mathcal{T}_{h}}\left(-C_{1} \ell(\mathbf{x}) \sqrt{k^{m}}\right)\left|\nabla u_{h}^{m}+\left(\nabla u_{h}^{m}\right)^{t}\right|^{2} \frac{k_{h}^{m+1}}{k_{h}^{m}} w_{h}|r| \pi d r d z \\
+\int_{\mathcal{T}_{h}} \frac{C_{3}}{\ell(\mathbf{x})} \sqrt{k_{h}^{m}} k_{h}^{m+1} w_{h}|r| \pi d r d z=0,
\end{array}\right.
$$

where $X_{h}^{m}(x)$ is a numerical approximation of $X^{m}(x)$. The parameters $C_{i}(\mathrm{i}=1,2$, $3)$ are adimentionalized constants.

The penalization term in equation (96)

$$
\int_{\mathcal{T}_{h}} p_{h}^{m+1} q_{h} \alpha_{0}|r| \pi d r d z
$$

with $\alpha_{0}=10^{-7}$, leads to a more regular problem [13]. 
The initial values for the velocity and pressure unknowns $\left(\mathbf{u}_{h}^{0}, p_{h}^{0}\right)$ are obtained by solving an auxiliary Stokes problem, and the turbulent kinetic energy $k_{h}^{0}$ is initialized to a constant in the entire domain.

The solving process is iterative. As soon as the final time $T$ is not reached, one solves numerically the kinetic energy problem, then the Navier-Stokes/Brinkman with eddy viscosity part, the time step is increased, the kinetic energy part is solved again and so on.

\subsection{Parameters settings}

Different parameters have to be set to perform the simulations:

- the parameter $\ell(\mathbf{x})$ in the definition of the eddy viscosity function (see equation (14)) is defined as a constant in each triangle, its value in a triangle being equal to the longest edge of this triangle,

- the water kinematic viscosity $\nu_{0}$ in equation (see equation (14)): $\nu_{0}=1.141 \times 10^{-6}$ $\mathrm{m}^{2} \mathrm{~s}^{-1}$ at $15^{\circ} \mathrm{C}$,

- the initial turbulent kinetic energy equal to a constant in the entire domain and equal to $0.01 \mathrm{~m}^{2} \mathrm{~s}^{-2}$,

- the permeability $K$ in the different regions:

$$
K(\mathbf{x})=\left\{\begin{array}{l}
10^{4} \mathrm{~s} \text { in the fluid region } \Omega_{w} ; \\
10^{-6} \mathrm{~s} \text { in the catch region } G_{c} \text { and collar region } G_{f} ; \\
1 \mathrm{~s} \text { in the net region } G_{n}^{1} \\
5 \mathrm{~s} \text { in the net region } G_{n}^{2} ; \\
6 \mathrm{~s} \text { in the net region } G_{n}^{3} .
\end{array}\right.
$$

The unit of $K$ is $[\mathrm{s}]$ since it is formally the ratio between a permeability surface $\left[\mathrm{m}^{2}\right]$ under the kinematic viscosity $\left[\mathrm{m}^{2} \mathrm{~s}^{-1}\right]$.

Simulations have shown that the subdomain $G_{n}^{3}$ could be considered as permeable (i.e. as a fluid part). In fact, the mesh opening in $G_{n}^{3}$ is so high that the meshes do not disturb the flow.

- the time step set equal to $0.667 \mathrm{~s}$, 
- the adimentionalized constants, found numerically: $C_{1}=0.1 ; C_{2}=0.05 ; C_{3}=$ 0.03 .

\subsection{Numerical results}

Using the parameters defined in the previous section, we use the free software FreeFem++ to compute the fluid problem. Runs were made on a bi-processor Pentium Xeon EM64T 3.2Ghz, with 2Go RAM.

The global behavior of the flow is shown on Fig. 10 where the streamlines are drawn.

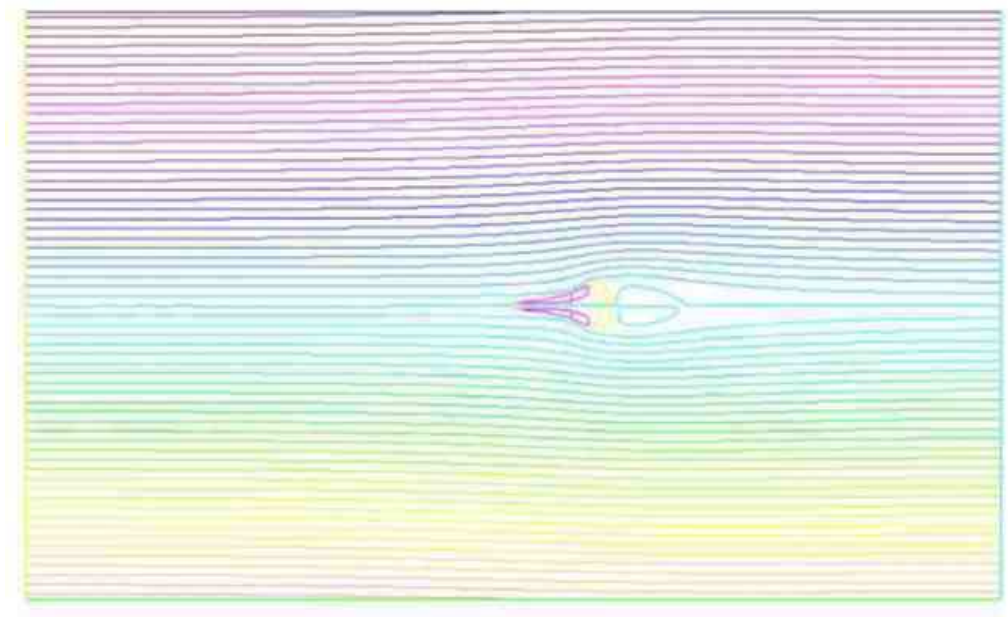

Fig. 10. Streamlines

The level curves of the $\mathrm{z}$ component of the mean velocity are given in Fig. 11.

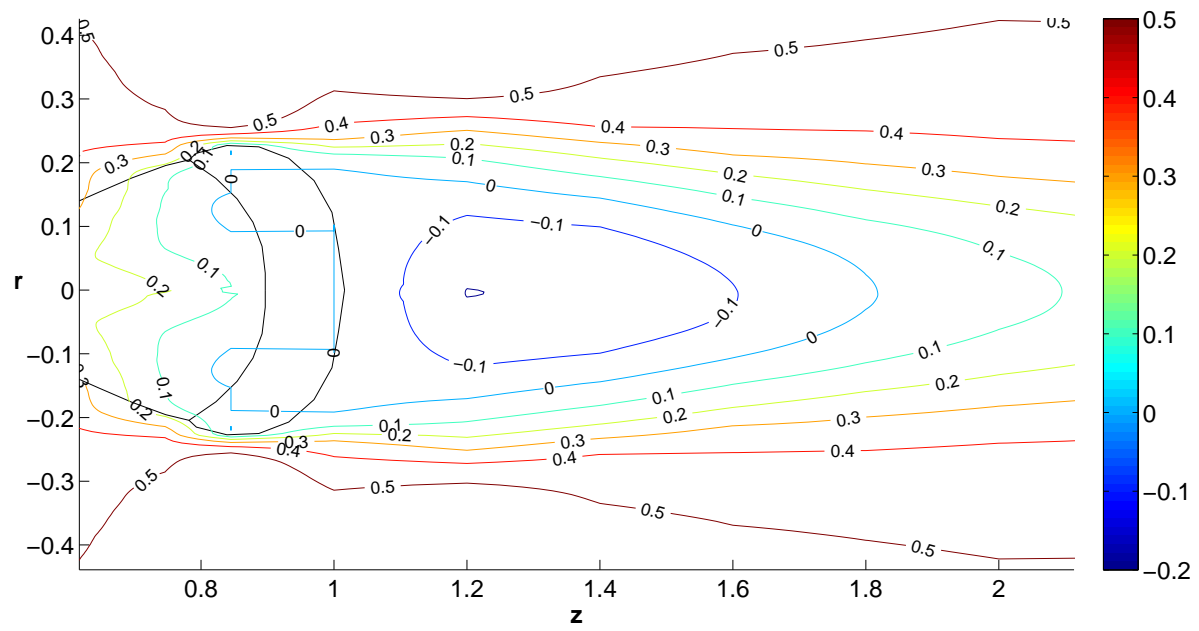

Fig. 11. Level curves of $u_{z}$ behind the catch

Fig. 12 gives the level curves of $u_{r}$ and the Fig. 13 and 14 gives those for the turbulent 
kinetic energy $k$.

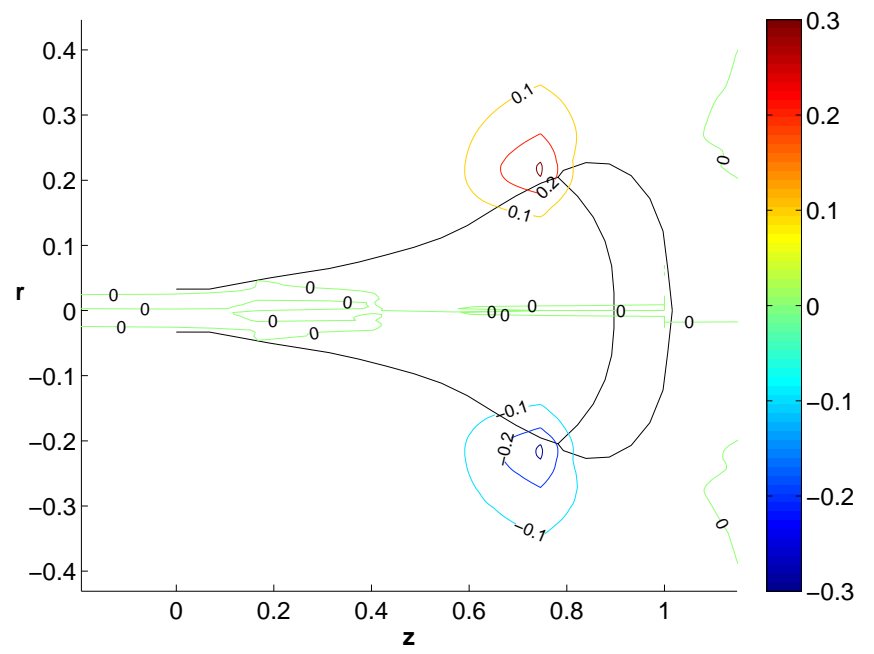

Fig. 12. Level curves for $u_{r}$

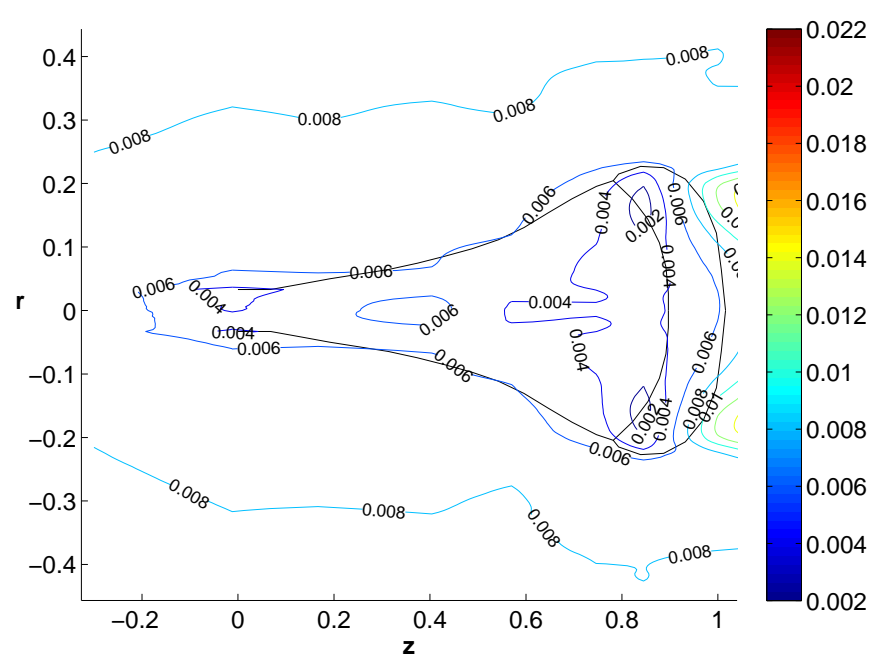

Fig. 13. Level curves for $k$ in the surroundings of the net

The use of an unstructured mesh leads to a slight asymmetry in the graphics for the turbulent kinetic energy.

Those figures give several results:

- a laminar flow at the output (see Fig. 10). It allows us to keep the simplified boundary conditions (80) - (81) at the output.

- the escapement of the inner velocity inside the net takes place just in front of the catch (see Fig. 12), 


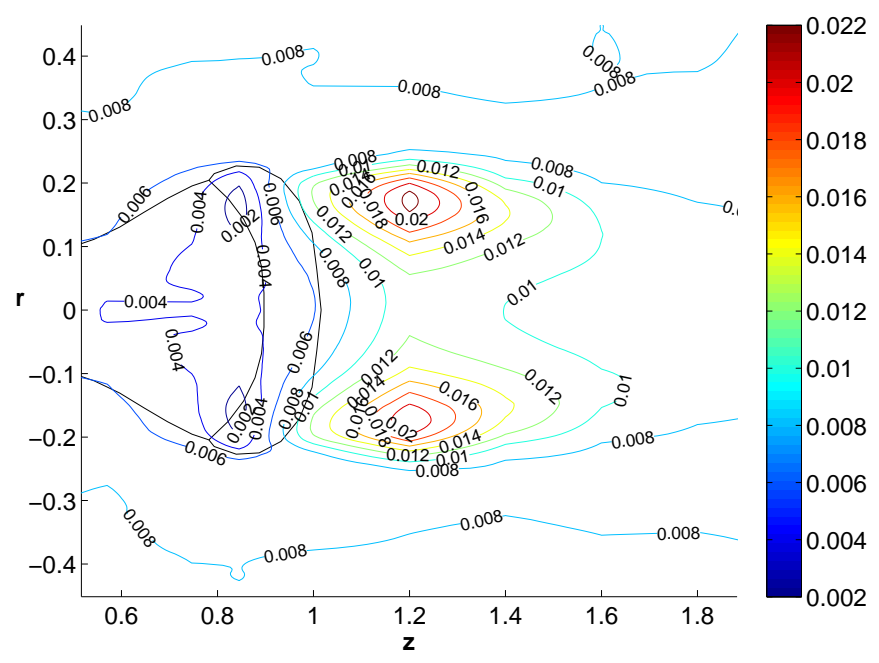

Fig. 14. Level curves for $k$ behind the catch

- the turbulence is mainly located behind the catch and is low in the surroundings of the net (see Fig. 13 and 14),

- two main eddies are located behind the catch (see Fig. 10, 11, 14).

Remark 6.1 Notice the axisymmetry hypothesis of the flow prevents any vortex shedding. Such an hypothesis is reasonable in the case of the rigid net model to predict the velocity profiles near the net. However it would no longer be valid in the fully $3 D$ case with a moving net.

Let us compare now the experimental profiles given at the beginning (Fig. 3), measured by a LDV technique for $u_{z}$ with those obtained numerically (see Fig. 15-16-17). 

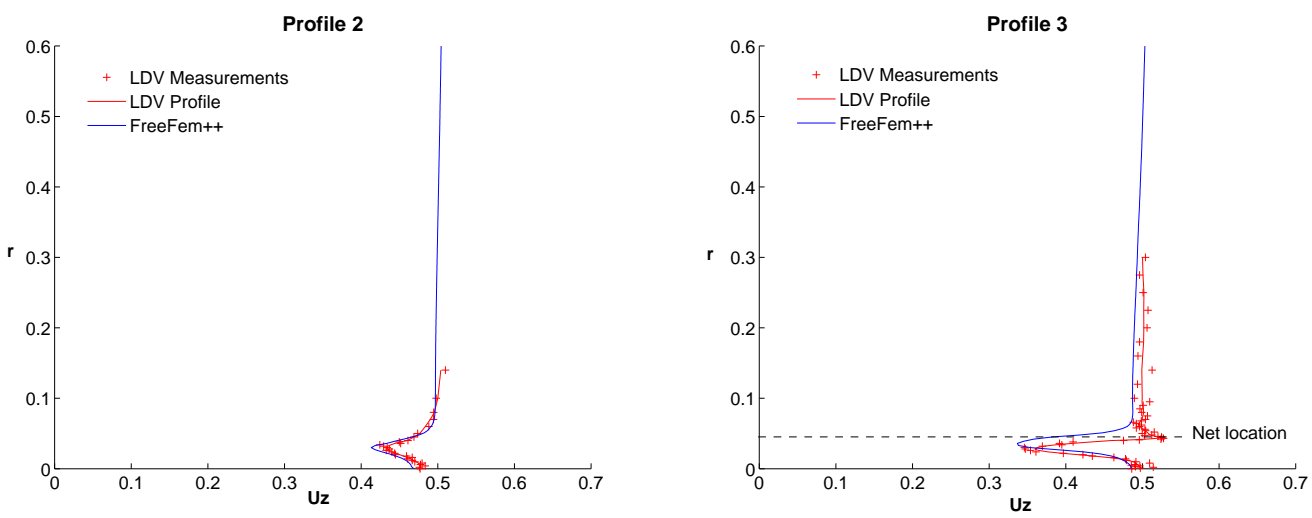

Fig. 15. Profiles 2 and 3 after 50 iterations
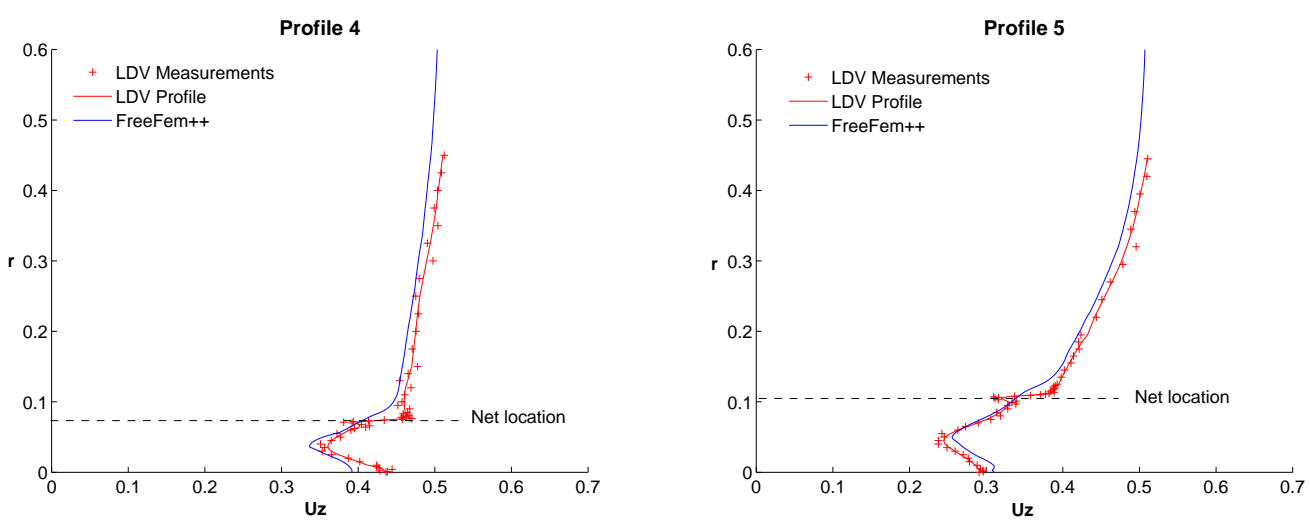

Fig. 16. Profiles 4 and 5 after 50 iterations
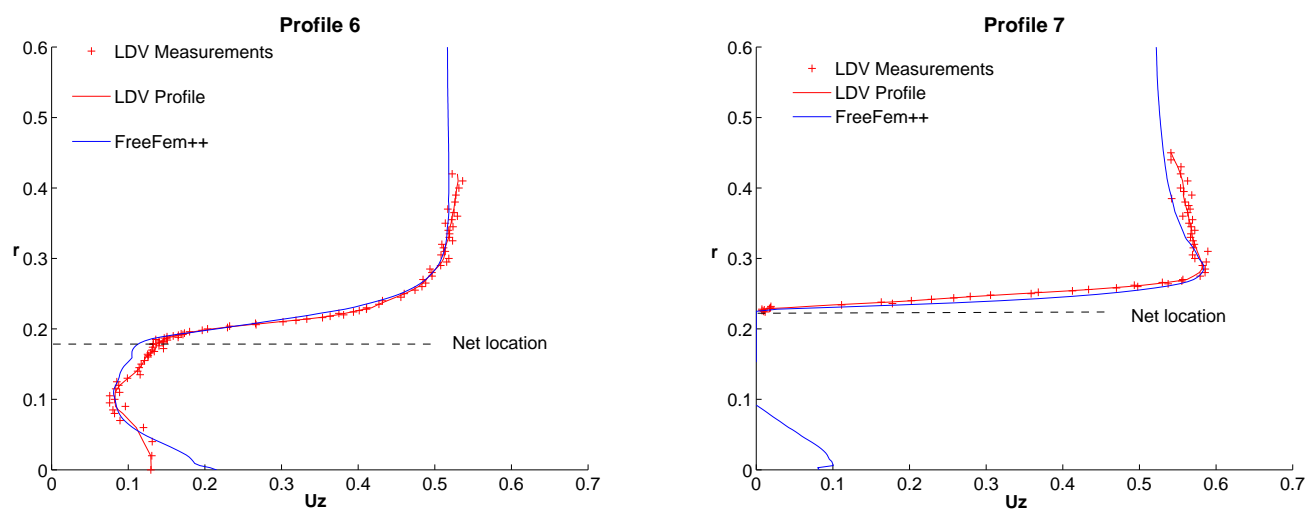

Fig. 17. Profiles 6 and 7 after 50 iterations

One can see that the numerical profiles fit well with those obtained experimentally (see Fig. 15-16-17).

An interesting feature is emphasized by computing the norm 2 of the difference of the velocity and the turbulent kinetic energy between two successive iterations (see Fig. 18). A stationary state is reached after about 50 iterations: the residual for $\mathbf{u}$ is equal to $10^{-3}$, and the one for $k$ equal to $4 \times 10^{-4}$. This is in agreement with the fact that we are studying mean quantities. 

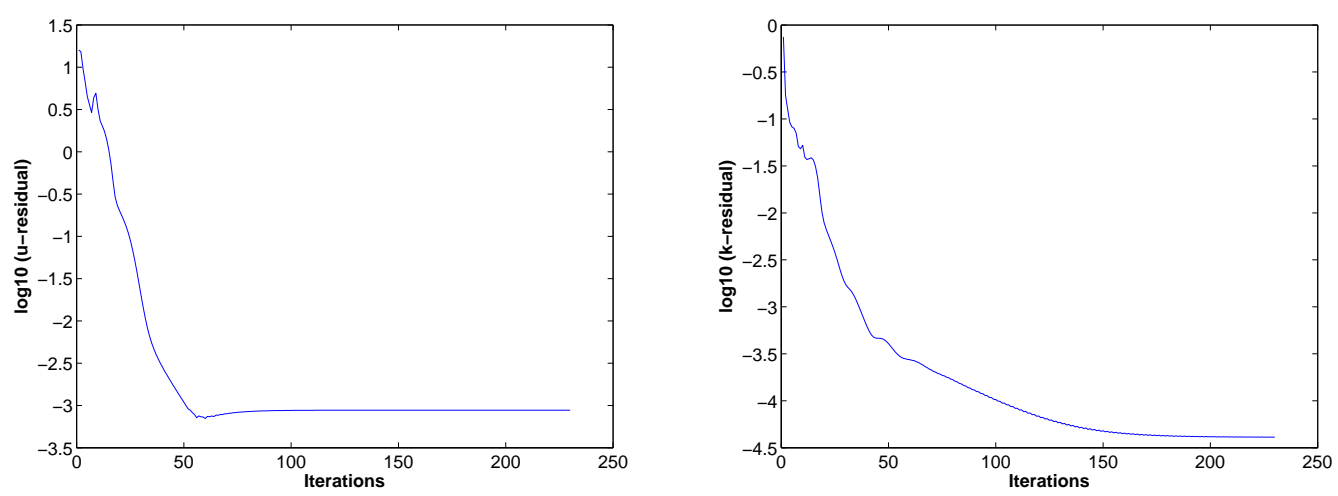

Fig. 18. $\log 10$ of the residual computed for the velocity and TKE vs iterations

To conclude, we have a model that leads to remarkable results in comparison with the available experimental data. In this particular case of a rigid net, our model looks appropriate. Moreover, this model has the advantage that its application to a $3 \mathrm{D}$ problem is possible, especially if we make use of a fictitious domain technique that does not require a complex mesh generation.

Acknowledgements: We thank IFREMER and the region Bretagne for the financial support of this work. We are very grateful toward F. Hecht for fruitful discussions about the numerical simulation of our problem. We also thank G. Germain and his team of the IFREMER center of Boulogne-sur-Mer for the experimental data, B. Vincent of the IFREMER center of Lorient (France) for his relevant remarks and D. Priour of the IFREMER center of Brest (France). Finally we express our grateful gratitude to D. Madden who has reread this paper and corrected the english.

\section{References}

[1] G. Allaire, Homogenization of the Navier-Stokes equations and derivation of Brinkman's law, Applied Mathematics for Engineering Sciences, C. Carasso et al. eds., Cépaduès Editions, Toulouse, 7-20, 1991.

[2] P. Angot, C.H. Bruneau, P. Fabrie, A penalization method to take into account obstacles in viscous flows, Numer. Math., Vol. 81, 497-520, 1999.

[3] L. Botsford, J. Castilla, C. Peterson, The management of fisheries and marine ecosystems, Science, Vol. 277, 509-515, 1997.

[4] L. Boccardo, T. Gallouët, Nonlinear elliptic and parabolic equations involving measure data, J. Funct. Anal., Vol. 87, 149-169, 1989.

[5] G. -K. Batchelor, An introduction to fluid dynamics, Cambridge university press, 1973.

[6] H. Brézis, Analyse fonctionnelle, seconde édition, Masson, 1993.

[7] C. -H. Bruneau, P. Fabrie, New efficient boundary conditions for incompressible Navier-Stokes equations: a well-posedness result, RAIRO Modél. Math. Anal. Numér., Vol. 30, 815-840, 1996. 
[8] M. Dauge, C. Bernardi, Y. Maday, Spectral methods for axisymmetric domains, Gauthier Villars, 1999.

[9] M. Dauge, C. Bernardi, Y. Maday, Polynomials in the Sobolev World, Publications du Laboratoire J. -L. Lions, 2003.

[10] G. Germain, J. V. Facq, D. Priour, Flow characterization around a cod-end, IMAM congress, Portugal, 2005.

[11] V. Girault, P-A. Raviart, Finite Element Methods for Navier-Stokes Equations, Springer-Verlag, 1986.

[12] P. Grisvard, Singularités des solutions du problème de Stokes dans un polygone, Univ. de Nice, 1978.

[13] F. Hecht, O. Pironneau, A. Le Hyaric, K. Ohtsua, FreeFem++ Manual, Laboratoire Jacques Louis Lions, Paris (http://freefem.org/ff++), 2006.

[14] K. Khadra, P. Angot, S. Parneix, J.P. Caltagirone, Fictitious domain approach for numerical modelling of Navier-Stokes equations, International journal for numerical methods in fluids, Vol. 34, 651-684, 2000.

[15] J. Lederer, R. Lewandowski, On the RANS 3D model with unbounded eddy viscosities, Ann. IHP an. non lin., to appear, see also at http://perso.univrennes1.fr/roger.lewandowski/, 2006.

[16] R. Lewandowski, The mathematical analysis of the coupling of a turbulent kinetic energy equation to the Navier-Stokes equation with an eddy viscosity, Nonlinear Analysis TMA, Vol. 28 (number 2), 393-417, 1997.

[17] R. Lewandowski, Analyse Mathématique et Océanographie, Masson, 1997.

[18] R. Lewandowski, Vorticities in a LES model for 3D periodic turbulent flows, Journ. of Math. Fluid Mech., Vol. 8, 398-422, 2006.

[19] H. Le Dret, R. Lewandowski, D. Priour, F. Chagneau, Numerical Simulation of a Cod End Net. Part 1: Equilibrium in a Uniform Flow, Journal of Elasticity, Vol. 76, 139-162, 2004.

[20] J. -L. Lions, E. Magenes, Problèmes aux limites non homogènes et application, volume 1, Dunod, 1968.

[21] R. Mittal, G. Iaccarino, Immersed boundary methods, Annual Rev. Fluid Mech., Vol. 37, 239-261, 2005.

[22] B. Mohammadi, O. Pironneau, Analysis of the k-epsilon turbulence model, Masson, Springer, 1994.

[23] F. Murat, Solutiones renormalizadas de EDP elipticas no lineales, Lectures at Sevilla's University, 1990.

[24] F. -G. O'Neill, Axisymmetric trawl cod-ends made from netting of a general mesh shape, IMA J. Appl. Math., Vol. 62, 245-262, 1999.

[25] C. -S. Peskin, Flow patterns around heart valves: a numerical method, Journal of computational physics, Vol. 10, 1972.

[26] G. Pichot, Modélisation et analyse numérique du couplage filet-écoulement hydrodynamique en vue d'estimer la forme de la prise dans une poche chalut, $\mathrm{PhD}$ Thesis of Rennes 1 University, to be defended, 2007.

[27] D. Priour, Calculation of net shapes by the finite element method with triangular elements, Comm. in Num. Meth., Vol. 15, 755-763, 1999.

[28] B. Vincent, Etude numérique et expérimentale des écoulements guidés par une paroi perméable axisymétrique. Application à la modélisation des chaluts pour 
en améliorer la sélectivité, Thèse Ecole Centrale Nantes, 1996.

[29] G. Stampacchia, Équations elliptiques du second ordre à coefficients discontinus, Les presses de l'université de Montréal, 1966.

\section{Appendix - Proofs of Lemmas and Proposition}

Here are given the proofs of the technical lemmas 5.1 and 5.2 and of Proposition 5.1 .

- Lemma 5.1. Let

$$
V=\left\{\mathbf{v} \in\left(H^{1}(\Omega)\right)^{2} ; \quad \nabla \cdot \mathbf{v}=0 ;\left.\quad \mathbf{v}\right|_{\Gamma_{i} \cup \Gamma_{l}}=\mathbf{0} .\right\}
$$

and

$$
\mathcal{V}=\left\{\mathbf{v} \in\left[C^{\infty}(\Omega)\right] ; \exists \mathbf{v} \in \tilde{\mathcal{V}} \text { s.t. } \mathbf{v}=\mathbf{v}_{r}\right\}
$$

The space $\mathcal{V}$ is dense in $V$.

Proof. Let $\mathbf{v} \in V$. Recall we denote:

$$
\begin{gathered}
\tilde{V}=\left\{\mathbf{v} \in\left(H^{1}(\tilde{\Omega})\right)^{2} ; \quad \nabla \cdot \mathbf{v}=0 ;\left.\quad \mathbf{v}\right|_{\partial \tilde{\Omega}}=\mathbf{0}\right\} \\
\tilde{\mathcal{V}}=\left\{\mathbf{v} \in(\mathcal{D}(\tilde{\Omega}))^{2} ; \quad \nabla \cdot \mathbf{v}=0\right\} .
\end{gathered}
$$

Since $\tilde{\Omega}$ is simply connected and has a Lipschitz boundary, one knows thanks to Corollary 2.5 in [11] that $\tilde{\mathcal{V}}$ is dense in $\tilde{V}$. Therefore, there exists a sequence $\left(\mathbf{w}_{n}\right)_{n \in \mathbf{N}}$ of fields in $\tilde{\mathcal{V}}$ that converges to $\mathbf{v}^{e}$ in the space $\tilde{V}$. One obviously has

$$
\int_{\Omega}\left|\nabla\left(\left(\mathbf{w}_{n}\right)_{r}-\mathbf{v}\right)\right|^{2} \leq \int_{\Omega^{s}}\left|\nabla\left(\mathbf{w}_{n}-\mathbf{v}^{e}\right)\right|^{2} .
$$

This shows that the sequence $\left(\left(\mathbf{w}_{n}\right)_{r}\right)_{n \in \mathbb{N}}$ converges to $\mathbf{v}$ in $V$ and each $\left(\mathbf{w}_{n}\right)_{r}$ lies in $\mathcal{V}$ by definition. The lemma is proven.

- Lemma 5.2. Let

$$
W=L^{2}([0, T], V) \cap L^{\infty}\left([0, T],\left(L^{2}(\Omega)\right)^{2}\right.
$$

and $(\tilde{\mathbf{u}}, \mathbf{v}) \in W \times W$. Then

$$
\int_{0}^{T} \int_{\Gamma_{o}}[\tilde{\mathbf{u}} . \mathbf{n}]^{-} \tilde{\mathbf{u}} \cdot \mathbf{v} \leq C\|\tilde{\mathbf{u}}\|_{W}^{2}\|\mathbf{v}\|_{W}
$$

where $C$ is a constant that only depends on $\alpha$ and $\beta$. Moreover, there also exists a constant $\tilde{C}$ such that

$$
\forall \tilde{\mathbf{v}} \in L^{8 / 3}([0, T], V), \quad \int_{0}^{T} \int_{\Gamma_{o}}[\tilde{\mathbf{u}} . \mathbf{n}]^{-} \tilde{\mathbf{u}} \cdot \mathbf{v} \leq C\|\tilde{\mathbf{u}}\|_{W}^{2}\|\tilde{\mathbf{v}}\|_{L^{8 / 3}([0, T], V)}
$$


Proof. Let $\mathbf{v} \in W$. On starts from the classical interpolation inequality (see in $[20])$

$$
\|\mathbf{v}\|_{H^{3 / 4}} \leq\|\mathbf{v}\|_{L^{2}}^{1 / 4}\|\mathbf{v}\|_{H^{1}}^{3 / 4}
$$

One deduces that

$$
\|\mathbf{v}\|_{L^{8 / 3}\left(H^{3 / 4}\right)} \leq\|\mathbf{v}\|_{L^{\infty}\left(L^{2}\right)}^{1 / 4}\|\mathbf{v}\|_{L^{2}(V)}^{3 / 4} \leq \frac{1}{4}\|\mathbf{v}\|_{L^{\infty}\left(L^{2}\right)}+\frac{3}{4}\|\mathbf{v}\|_{L^{2}(V)} \leq\|\mathbf{v}\|_{W} .
$$

One deduces by the trace Theorem that

$$
\|\mathbf{v}\|_{L^{8 / 3}\left(H^{1 / 4}\left(\Gamma_{o}\right)\right)} \leq C\|\mathbf{v}\|_{W} .
$$

Moreover, thanks to the Sobolev Theorem,

$$
\|\mathbf{v}\|_{L^{8 / 3}\left(L^{4}\left(\Gamma_{o}\right)\right)} \leq C\|\mathbf{v}\|_{W}
$$

Let $\tilde{\mathbf{u}} \in W$. It is clear that at $\Gamma_{0}$,

$$
\tilde{\mathbf{u}} . \mathbf{n} \in L^{\infty}\left(H^{-1 / 2}\left(\Gamma_{0}\right)\right) \cap L^{2}\left(H^{1 / 2}\left(\Gamma_{0}\right)\right) .
$$

By using again a simple interpolation inequality one deduces easily that

$$
\|\tilde{\mathbf{u}} . \mathbf{n}\|_{L^{4}\left(L^{2}\left(\Gamma_{o}\right)\right)} \leq C\|\tilde{\mathbf{u}}\|_{W} .
$$

Therefore, $(\tilde{\mathbf{u}} . \mathbf{n}) \tilde{\mathbf{u}} \in L^{8 / 5}\left(L^{4 / 3}\left(\Gamma_{0}\right)\right)$, as well as $(\tilde{\mathbf{u}} . \mathbf{n})^{-} \tilde{\mathbf{u}}$ and one has

$$
\left\|(\tilde{\mathbf{u}} . \mathbf{n})^{-} \tilde{\mathbf{u}}\right\|_{L^{8 / 5}\left(L^{4 / 3}\left(\Gamma_{0}\right)\right)} \leq C\|\tilde{\mathbf{u}}\|_{W}^{2} .
$$

The rest of the proof is now a direct consequence of (104), (106) and Hölder inequality.

- Proposition 5.1 There exists a constant $C_{1}=C_{1}\left(\mathbf{u}_{0}, u_{I}, \nu, \alpha, \beta\right)$ and for each $p<4 / 3$ a constant $C_{2}=C_{2}\left(\mathbf{u}_{0}, u_{I}, \nu, \mu, p, \alpha, \beta\right)$ such that for any smooth solution $(\tilde{\mathbf{u}}, k)$ to the variational problem $[(67),(66)]$ one has

$$
\begin{aligned}
& \|\tilde{\mathbf{u}}\|_{L^{2}([0, T], V)}+\|\tilde{\mathbf{u}}\|_{L^{\infty}\left([0, T], L^{2}(\Omega)\right)} \leq C_{1}, \\
& \|k\|_{L^{p}\left([0, T], W^{1, p}(\Omega)\right)} \leq C_{2} .
\end{aligned}
$$

Proof. We proceed in two steps. We first estimate the velocity and then the Turbulent Kinetic Energy (TKE).

Step 1. Estimating the velocity. One multiplies the equation (51) by $\tilde{\mathbf{u}}$ and integrates on $\Omega$. A technical but easy computation using the boundary condition $\tilde{\mathbf{u}}$ (56) yields:

$$
\begin{aligned}
& \frac{1}{2} \frac{d}{d t}\|\tilde{\mathbf{u}}\|_{L^{2}(\Omega)}^{2}+\int_{\Omega} 2 \nu_{t}(k)|\varepsilon(\tilde{\mathbf{u}})|^{2}+\int_{\Omega} 2 \nu_{t}(k) \boldsymbol{\varepsilon}(\tilde{\mathbf{u}}) \boldsymbol{\varepsilon}\left(\mathbf{v}_{0}\right)+ \\
& \int_{\Omega} \mathcal{P}(\tilde{\mathbf{u}}) \cdot \tilde{\mathbf{u}}-\int_{\Omega} \mathbf{v}_{0} \otimes\left(\tilde{\mathbf{u}}+\mathbf{v}_{0}\right): \nabla \tilde{\mathbf{u}}+\frac{1}{2} \int_{\Gamma_{0}}\left(\left(\tilde{\mathbf{u}}+\mathbf{v}_{0}\right) \cdot \mathbf{n}\right)^{+}|\tilde{\mathbf{u}}|^{2}=0 .
\end{aligned}
$$


Since

$$
0 \leq \int_{\Omega} \mathcal{P}(\tilde{\mathbf{u}}) \cdot \tilde{\mathbf{u}} \quad \text { and } \quad 0 \leq \frac{1}{2} \int_{\Gamma_{0}}\left(\left(\tilde{\mathbf{u}}+\mathbf{v}_{0}\right) \cdot \mathbf{n}\right)^{+}|\tilde{\mathbf{u}}|^{2},
$$

using (22) and (46), the energy equality (111) yields

$$
\begin{aligned}
\frac{1}{2} \frac{d}{d t} \| \tilde{\mathbf{u}}||_{L^{2}(\Omega)}^{2}+\int_{\Omega} 2 \nu_{t}(k)|\varepsilon(\tilde{\mathbf{u}})|^{2} \leq & N \int_{\Omega}|\varepsilon(\tilde{\mathbf{u}})|\left|\varepsilon\left(\mathbf{v}_{0}\right)\right|+|| \mathbf{v}_{0} \|_{\infty} \int_{\Omega}|\tilde{\mathbf{u}}||\nabla \tilde{\mathbf{u}}| \\
& +\left\|\mathbf{v}_{0}\right\|_{\infty}^{2} \int_{\Omega}|\nabla \tilde{\mathbf{u}}|
\end{aligned}
$$

By using Young and Korn's inequalities, one has

$$
\begin{aligned}
& \int_{\Omega}|\varepsilon(\tilde{\mathbf{u}})|\left|\varepsilon\left(\mathbf{v}_{0}\right)\right| \leq \frac{1}{2 \zeta} \int_{\Omega}\left|\varepsilon\left(\mathbf{v}_{0}\right)\right|^{2}+\frac{\zeta}{2} \int_{\Omega}|\varepsilon(\tilde{\mathbf{u}})|^{2}, \\
& \int_{\Omega}|\tilde{\mathbf{u}}||\nabla \tilde{\mathbf{u}}| \leq\left(\frac{1}{2 \zeta}+C\right) \int_{\Omega}|\tilde{\mathbf{u}}|^{2}+\frac{\zeta}{2} C \int_{\Omega}|\varepsilon(\tilde{\mathbf{u}})|^{2} .
\end{aligned}
$$

where $\zeta$ will be fixed later on and $C$ is the constant in the Korn inequality. Finally, by always using the Young inequality combined with the Cauchy-Schwarz inequality,

$$
\left\|\mathbf{v}_{0}\right\|_{\infty}^{2} \int_{\Omega}|\nabla \tilde{\mathbf{u}}| \leq \frac{\alpha \beta}{2 \zeta}\left\|\mathbf{v}_{0}\right\|_{\infty}^{4}+\frac{\zeta}{2} \int_{\Omega}|\varepsilon(\tilde{\mathbf{u}})|^{2}
$$

Thereofore, (112) combined with (22) yields

$$
\begin{aligned}
\frac{d}{2 d t}\|\tilde{\mathbf{u}}\|_{L^{2}(\Omega)}^{2}+\left(\nu_{0}-(\zeta / 2)(N+C+1)\right) \int_{\Omega}|\varepsilon(\tilde{\mathbf{u}})|^{2} \leq & \left(\frac{1}{2 \zeta}+C\right)\|\tilde{\mathbf{u}}\|_{L^{2}(\Omega)}^{2} \\
& +\frac{N}{2 \zeta} \int_{\Omega}\left|\varepsilon\left(\mathbf{v}_{0}\right)\right|^{2} \\
& +\frac{\alpha \beta}{2 \zeta}\left\|\mathbf{v}_{0}\right\|_{\infty}^{4}
\end{aligned}
$$

We choose $\zeta$ be such that $\left(\nu_{0}-(\zeta / 2)(N+C+1)\right)=\nu_{0} / 2$. One deduces from (116) and Gronwall's lemma, combined again with Korn's inequality, the existence of $\tilde{C}=\tilde{C}\left(\mathbf{u}_{\mathrm{I}}, N, \nu_{0}, \alpha, \beta, T, \mathbf{u}_{0}\right)$, which blows up in a $e^{T}$ rate and such that

$$
\|\tilde{\mathbf{u}}\|_{W}=\|\tilde{\mathbf{u}}\|_{L^{\infty}\left([0, T], L^{2}(\Omega)\right)}+\|\tilde{\mathbf{u}}\|_{L^{2}([0, T], V)} \leq \tilde{C} .
$$

Step 2. Estimating the TKE. Notice first that by using the same arguments as in [16] or in [17], one can make sure that $k \geq 0$ a.e. as far as we assume $k_{0} \geq 0$. The boundary terms does not create any troubles because

$$
\int_{0}^{T} \int_{\Gamma_{o}}\left(\left(\tilde{\mathbf{u}}+\mathbf{v}_{0}\right) \cdot \mathbf{n}\right)^{-} k\left(-k^{-}\right)=\int_{0}^{T} \int_{\Gamma_{o}}\left(\left(\tilde{\mathbf{u}}+\mathbf{v}_{0}\right) \cdot \mathbf{n}\right)^{-}\left(k^{-}\right)^{2} \geq 0 .
$$

The other terms are like in the general situation studied in [17] chapter 4. From now and throughout the rest of the paper, one works with $k \geq 0$. 
Thanks to (117), we can use the Classical Boccardo-Gallouët estimate (see [4]). By a proof already done in [16], [17], [18] and [15] and since we are working in a $2 \mathrm{D}$ case and $k=0$ on $\partial \Omega$, one deduces that

$$
\exists \bar{C}=\bar{C}\left(\mathbf{u}_{\mathrm{I}}, N, \nu_{0}, \alpha, \beta, T, \mathbf{u}_{0}\right) ; \quad\|k\|_{L^{\infty}\left([0, T], L^{1}(\Omega)\right)} \leq \bar{C},
$$

and

$$
\forall p<4 / 3, \quad \exists \hat{C}=\hat{C}\left(p, \mathbf{u}_{\mathrm{I}}, N, \nu_{0}, \alpha, \beta, T, \mathbf{u}_{0}\right) ; \quad\|k\|_{L^{p}\left([0, T], W_{0}^{1, p}(\Omega)\right)} \leq \hat{C} .
$$

